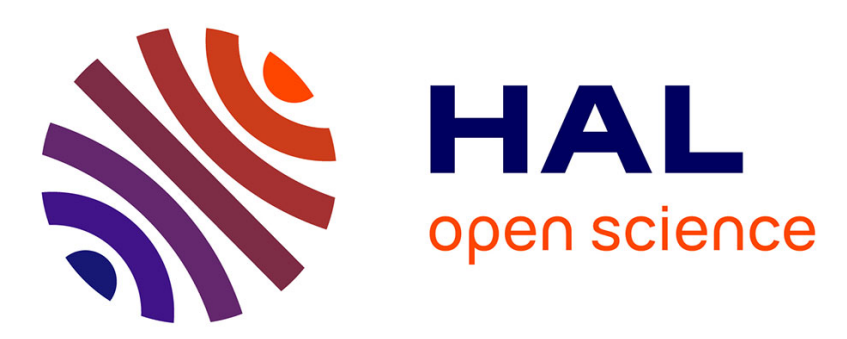

\title{
Hydrodynamic Coefficient Computation for a Partially Submerged Wave Energy Converter
}

Sébastien Olaya, Jean-Matthieu Bourgeot, Mohamed Benbouzid

\section{To cite this version:}

Sébastien Olaya, Jean-Matthieu Bourgeot, Mohamed Benbouzid. Hydrodynamic Coefficient Computation for a Partially Submerged Wave Energy Converter. IEEE Journal of Oceanic Engineering, 2015, 40 (3), pp.522-535. 10.1109/JOE.2014.2344951 . hal-01175747

\section{HAL Id: hal-01175747 \\ https://hal.science/hal-01175747}

Submitted on 12 Jul 2015

HAL is a multi-disciplinary open access archive for the deposit and dissemination of scientific research documents, whether they are published or not. The documents may come from teaching and research institutions in France or abroad, or from public or private research centers.
L'archive ouverte pluridisciplinaire HAL, est destinée au dépôt et à la diffusion de documents scientifiques de niveau recherche, publiés ou non, émanant des établissements d'enseignement et de recherche français ou étrangers, des laboratoires publics ou privés. 


\title{
Hydrodynamic Coefficient Computation for a Partially Submerged Wave Energy Converter
}

\author{
Sébastien Olaya, Jean-Matthieu Bourgeot, and Mohamed Benbouzid, Senior Member, IEEE
}

\begin{abstract}
This paper deals with the hydrodynamic parameter computation of a wave energy converter that consists of a cylindrical buoy sliding along a partially submerged platform made up of a plate and a column. The computed parameters are especially needed for the development of a simple hydrodynamic time-dependant model, based on the Cummins' formulation. This model is intended for WEC control purposes. A semi-analytical approach is proposed for the computation of the hydrodynamic coefficients and the excitation forces. The boundary value problem is solved by using variable separation and matched eigenfunction expansion methods. Analytical expressions for the velocity potential are then obtained for each sub-domain. The hydrodynamic coefficients and the excitation force can then be computed by using these expressions. Numerical results are given for different buoy, column, and plate radiuses and clearly the bearing surface of the plate has a significant influence on the wave excitation force applied to the submerged platform.
\end{abstract}

\section{Index Terms}

Wave energy converter, potential theory, eigenfunction expansion, wave-loads, heaving mode, scattering and radiation problem.

\section{INTRODUCTION}

This work was motivated by the need to provide a set of hydrodynamic parameters for the development of a simple hydrodynamic time-dependant model, based on the Cummins' formulation for a Wave Energy Converter (WEC) [1], [2]. This time domain model is needed to analyse the WEC behaviour in irregular waves. The WEC device schematically depicted on Fig. 1 consists of a cylindrical buoy floating on waves and sliding along a partially submerged platform made up of a plate and a column. Energy resulting from the relative motion between the two concentric bodies is extracted from the incoming wave by a power take-off (PTO) consisting of a gearbox and an electric generator. The present paper focuses on determining the excitation forces and hydrodynamic coefficients (i.e. added mass and radiation damping) in heaving mode and in water of a finite depth. It concludes the work

S. Olaya and J.-M. Bourgeot are at the École Nationale d'Ingénieurs de Brest, EA 4325 LBMS, 945, Avenue Technopôle, 29280 Plouzané, France (e-mail: olaya@enib.fr, bourgeot@enib.fr).

M.E.H. Benbouzid is at the Université de Bretagne Occidentale, EA 4325 LBMS, Rue de Kergoat, CS 93837, 29238 Brest Cedex 03, France (e-mail: Mohamed.Benbouzid@univ-brest.fr). 


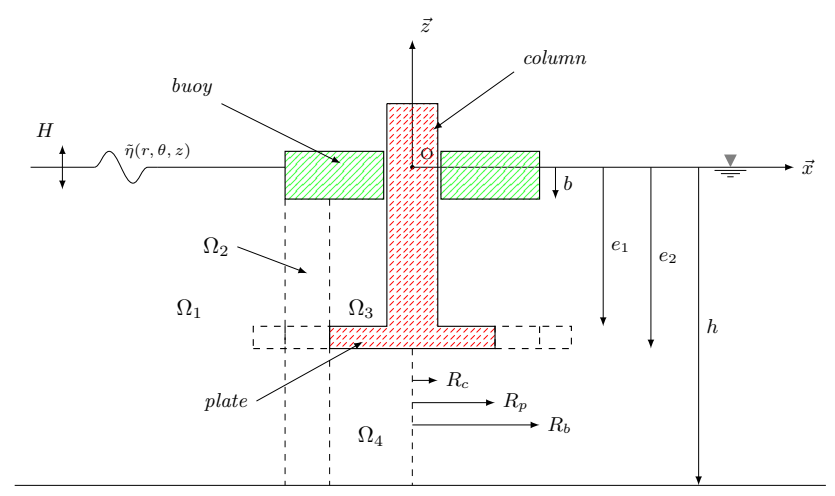

Fig. 1. Definition sketch of the wave energy converter.

introduced in [3] in which only part of the problem has been presented. Due to the simplicity of the geometry and in view of the model purposes (i.e. optimization and control) using a semi-analytical approach is probably the fastest and most reliable method to evaluate these parameters.

From the literature review, it has been noticed that in [4], Berggren and Johansson (1992) have studied the radiation problem of a wave energy device consisting of a buoy connected to a submerged plate by an elastomer hose. The buoy and plate have the same radius. They presented the results for added mass and potential damping for the two bodies and included cross terms only for the heaving mode. Under the assumption of linear wave theory, the radiation problem is solved using the matched eigenfunction expansion method. A comparison is made between their solution and a solution proposed by Yeung (1981) [5] who studied the whole radiation problem of a floating vertical cylinder following a method similar to that presented by Garrett (1971) in [6] who solves the scattering problem for a circular dock. In the case of a single cylinder, it is worth noting that a summary of both problems, i.e. radiation and scattering, can be found in [7] published by Bhatta and Rahman (2003) and for which numerical computation aspects and results are shown in [8]. Eidsmoen (1995) [9], studied the case where the buoy is rigidly connected to a submerged vertical tube in which a plate acts as a piston. In addition to the radiation problem, the author has presented results for the excitation forces obtained through the Haskind's theorem. In [10], Zheng et al. (2005) have extended the solution for the radiation problem of a two-body coaxial axisymmetric system by adding the sway/surge and roll/pitch modes of motion as well as the solution to the scattering of water waves using the velocity potential. As in [9] authors used Haskind's relation to check the correctness of their diffraction results and used the symmetry of the cross terms in the matrices for the added mass and radiation damping coefficients. In term of theoretical results, Willams et al. (2000) investigated in [11] the case for which the floating cylinder has a side wall which is porous over a portion of its draught. Fluid flow passing through this wall is assumed to follow Darcy's law. As previously mentioned, the buoy and plate radiuses are strictly the same. Wu et al. (2004) investigated in [12] hydrodynamic properties of a similar WEC where the buoy floating on waves is directly connected to a caisson fixed on the seabed by a rigid rope. Wave energy is harnessed by a liquid pump in the caisson through the rope. In this paper the caisson radius is greater than or equal to that of the buoy. In [13], the same authors extended the 
solution to the case where the caisson radius is smaller than that of the buoy. They presented analytical results for the responding amplitudes and hydrodynamic forces for different radius sizes of the submerged structure and the gap between them. It is shown that the buoy hydrodynamic behaviour becomes more complex when the caisson radius is large and only at a relatively low frequency. Also it seems that the resonance period does not vary regardless of the increase or decrease of the radius ratio and/or the gap. In [14], a similar study was performed by the same authors, with a two-body moving structure, but their analysis was restricted to the case where the submerged cylinder radius is larger than or equal to that of the floating one. A set of theoretical hydrodynamic parameters is presented and the behaviour is found to be similar to that of the previous study. More recently, some hydrodynamic results have been reported in [15] by Chau and Yeung (2012) and also in [16] by Cochet and Yeung (2012), for the UC Berkeley wave energy device. The main idea is to extract energy due to the relative heaving motion between two concentric surface-piercing truncated cylinders. A similar study was first performed by Mavrakos (2004) in [17].

Throughout the literature review, it is worth noting that there are no analytical results for the particular WEC configuration given in Fig. 1.

\section{Mathematical Formulation of the Problem}

Following the established procedure for this kind of resolution, we divided the whole fluid domain into three or four sub-domains depending on the plate radius size as indicated in Fig. 1. Considering a cylindrical coordinate system $(r, \theta, z)$ with its origin $O$ located at the intersection of the undisturbed free surface level with cylinder axes and the $z$-axis is positive upwards, it follows

- region $\Omega_{1}: r \geq R_{x} ;-h \leq z \leq 0$

where $R_{x}=\max \left(R_{b}, R_{p}\right)$

- region $\Omega_{2}$

- For $R_{p}>R_{b}: R_{b} \leq r \leq R_{p} ;-e_{1} \leq z \leq 0$

- For $R_{p}=R_{b}:$ does not exist

- For $R_{p}<R_{b}: R_{p} \leq r \leq R_{b} ;-h \leq z \leq-b$

- region $\Omega_{3}: R_{c} \leq r \leq R_{x} ;-e_{1} \leq z \leq-b$

where $R_{x}=\min \left(R_{b}, R_{p}\right)$

- region $\Omega_{4}: r \leq R_{p} ;-h \leq z \leq-e_{2}$

$R_{b}$ is the radius of the buoy, $R_{p}$ is the radius of the plate, $R_{c}$ is the radius of the column and $h$ is the not necessarily infinite depth of the water. Other geometrical parameters are given in Fig. 1.

Assuming linear wave theory and supposing that the fluid is inviscid, incompressible, and that its motion is irrotational, the fluid flow can be described, using a complex representation, by the velocity potential

$$
\Phi(r, \theta, z, t)=\operatorname{Re}\left\{\phi(r, \theta, z) e^{-\mathrm{i} \omega t}\right\}
$$


where $\operatorname{Re}\{\}$ denotes the real part of the complex expression, $\omega$ is the angular frequency and $t$ is the time dependency. Also let us define the free surface elevation as

$$
\eta(r, \theta, z, t)=\operatorname{Re}\left\{\tilde{\eta}(r, \theta, z) e^{-\mathrm{i} \omega t}\right\}
$$

which is related to the velocity potential by the linearised Free Surface Kinematic Boundary Condition (FSKBC)

$$
\frac{\partial \phi}{\partial z}=\frac{\partial \eta}{\partial t}, \quad \text { on } z=0
$$

and the linearised Free Surface Dynamic Boundary Condition (FSDBC)

$$
\frac{\partial \phi}{\partial t}+g \eta=0, \quad \text { on } z=0
$$

The spatial part of the velocity potential $\phi(r, \theta, z)$ has to satisfy the following boundary value problem

- Governing equation

$$
\Delta \phi=0, \quad \text { in the whole fluid domain }
$$

- Free surface boundary condition from (3) and (4)

- Seabed boundary condition

$$
\omega^{2} \phi-\left.g \frac{\partial \phi}{\partial z}\right|_{z=0}=0
$$

$$
\left.\frac{\partial \phi}{\partial z}\right|_{z=-h}=0
$$

- Body surface boundary condition

$$
\nabla \phi \cdot \vec{n}=\vec{U}_{k} \cdot \vec{n}, \quad \text { on } S_{k}, k=1,2
$$

where $g$ is the gravity acceleration, $\vec{n}$ the unit normal vector on wet body surfaces $S_{k}$ directed into the fluid domain, and $\vec{U}_{k}$ is the body velocity. $k=\{1,2\}$ stands for the buoy and the plate respectively. Finally, the velocity potential $\phi$ must satisfy the conditions which specify that the wave propagates away from the structure. This is given by the Sommerfeld radiation condition expressed in $\Omega_{1}$ as

$$
\lim _{r \rightarrow+\infty} \sqrt{r}\left(\frac{\partial \phi}{\partial r}-i k_{e} \phi\right)=0
$$

where $k_{e}$ is the wave number.

Based on the linear water wave theory and according to [1], the velocity potential can be broken down as follows

$$
\phi(r, \theta, z)=\phi_{0}(r, \theta, z)+\phi_{7}(r, \theta, z)+\sum_{k=1}^{2} \sum_{q=1}^{6} \phi_{q}^{k}(r, \theta, z)
$$

where $\phi_{0}$ is the incident waves potential, $\phi_{7}$ is the diffracted potential, and $\phi_{q}^{k}$ is the radiated potential due to the motion of the body $k$ in the direction $q$, with $q=\{1,3,5\}$ standing respectively for the surge, heave, and pitch mode of motion.

In the solution procedure, analytical expressions for velocity potentials are obtained using the variable separation method. Assuming angular independence due to the axisymmetric configuration, velocity potentials are expressed as infinite series of orthogonal functions into each sub-domain fulfilling all boundary conditions. More details can be found on mathematical formulations in [1], [18], [19]. 


\section{A. Radiated Potential for Each Sub-domain in Heaving Mode}

The radiation problem corresponds to the case where the structure is forced to oscillate in the absence of incident waves. Following a procedure similar to the one presented in [17], we can break down the velocity potential in each sub-domain, $\phi_{q}^{l, k}(r, \theta, z)$ as follows

$$
\phi_{q}^{l, k}(r, \theta, z)=\sum_{m=0}^{\infty}-\mathrm{i} \omega \zeta_{q} \varphi_{q, m}^{l, k}(r, z) \cos (m \theta)
$$

where $l$ denotes the fluid domain and $\zeta_{q}$ is the complex amplitude corresponding to the motion mode. In heaving mode $(q=3$ ) only expressions for $m=0$ gave a contribution [7]. We are then looking for solutions that can be expressed as

$$
\varphi_{3,0}^{l, k}(r, z)=\varphi_{3, h}^{l, k}(r, z)+\varphi_{3, p}^{l, k}(r, z)
$$

where $\varphi_{3, p}^{l, k}(r, z)$ represents a particular solution of the velocity potential in heaving mode that fulfils the inhomogeneous boundary conditions and $\varphi_{3, h}^{l, k}(r, z)$ is the homogeneous part of the solution to the boundary value problem. In region $\Omega_{1}$, the velocity potential can be expressed as

$$
\varphi_{3, h}^{1, k}(r, z)=A_{0}^{k} \frac{H_{0}^{(1)}\left(k_{e} r\right)}{H_{0}^{(1)}\left(k_{e} R_{x}\right)} Z_{0}^{1}(z)+\sum_{i=1}^{\infty} A_{i}^{k} \frac{K_{0}\left(\lambda_{i} r\right)}{K_{0}\left(\lambda_{i} R_{x}\right)} Z_{i}^{1}(z)
$$

where $R_{x}=\max \left(R_{b}, R_{p}\right) . A_{i}^{k}$ are the unknown Fourier coefficients, $H_{0}^{(1)}()$ are Hankel functions and $K_{0}^{(1)}()$ are modified Bessel functions of the second kind.

For convenience, we define $\lambda_{0}=-\mathrm{i} k_{e}$ then the dispersion relation is given in terms of the eigenvalues $\lambda_{i}$ by

$$
\omega^{2}=k_{e} g \tanh \left(k_{e} h\right)=-\lambda_{i} g \tan \left(\lambda_{i} h\right)
$$

and the depth dependency function $Z_{i}^{1}(z)$ is

$$
Z_{i}^{1}(z)=N_{\lambda_{i}}^{-1 / 2} \cos \left(\lambda_{i}(z+h)\right)
$$

where

$$
N_{\lambda_{i}}=\frac{1}{2}\left[1+\frac{\sin \left(2 \lambda_{i} h\right)}{2 \lambda_{i} h}\right]
$$

for $i=0,1, \cdots, \infty$

If region $\Omega_{2}$ is defined, for the velocity potential we have

$$
\varphi_{3, h}^{2, k}(r, z)=\sum_{l=0}^{\infty}\left[B_{l}^{k} S_{l}(r)+C_{l}^{k} \tilde{S}_{l}(r)\right] Z_{l}^{2}(z)
$$

Expressions for the functions $S_{l}(r), \tilde{S}_{l}(r)$ are defined in Appendix A in terms of modified Bessel functions of the first and second kind, and $B_{l}^{k}, C_{l}^{k}$ are the unknown Fourier coefficients. The depth dependency function $Z_{l}^{2}(z)$ is defined as

- In the case where $R_{p}>R_{b}$

$$
Z_{l}^{2}(z)=N_{\gamma_{l}}^{-1 / 2} \cos \left(\gamma_{l}\left(z+e_{1}\right)\right)
$$


where the dispersion relation is given in terms of eigenvalues $\gamma_{l}$ by

$$
\omega^{2}=k_{i} g \tanh \left(k_{i} e_{1}\right)=-\gamma_{l} g \tan \left(\gamma_{l} e_{1}\right)
$$

and

$$
N_{\gamma_{l}}=\frac{1}{2}\left[1+\frac{\sin \left(2 \gamma_{l} e_{1}\right)}{2 \gamma_{l} e_{1}}\right]
$$

for $l=0,1, \cdots, \infty$

From the body surface condition (8) we have

$$
\left.\frac{\partial \varphi_{3}^{2, k}}{\partial z}\right|_{z=-e_{1}}=\delta_{2 k}, \text { for } R_{b} \leq r \leq R_{p}
$$

and then

$$
\varphi_{3, p}^{2, k}(r, z)=\left(z+\frac{g}{\omega^{2}}\right) \delta_{2 k}
$$

where $\delta_{i j}$ is the Kronecker's delta defined as $\delta_{i j}=1$ if $i=j$, and $\delta_{i j}=0$ otherwise.

- In the case where $R_{p}<R_{b}$

$$
Z_{l}^{2}(z)= \begin{cases}1, & \text { for } l=0 \\ \sqrt{2} \cos \left(\gamma_{l}(z+h)\right), & \text { for } l \geq 1\end{cases}
$$

with

$$
\gamma_{l}=\frac{l \pi}{h-b}
$$

for $l=1,2, \cdots, \infty$.

From the body surface condition (8) we have

$$
\left.\frac{\partial \varphi_{3}^{2, k}}{\partial z}\right|_{z=-b}=\delta_{1 k}, \text { for } R_{p} \leq r \leq R_{b}
$$

and then

$$
\varphi_{3, p}^{2, k}(r, z)=\frac{1}{2(h-b)}\left[(z+h)^{2}-\frac{r^{2}}{2}\right] \delta_{1 k}
$$

In region $\Omega_{3}$, the velocity potential can be expressed as

$$
\varphi_{3, h}^{3, k}(r, z)=\sum_{n=0}^{\infty}\left[D_{n}^{k} T_{n}(r)+E_{n}^{k} \tilde{T}_{n}(r)\right] Z_{n}^{3}(z)
$$

Expressions for the functions $T_{n}(r), \tilde{T}_{n}(r)$ are defined in Appendix B in terms of modified Bessel functions of the first and second kind, and $D_{n}^{k}, E_{n}^{k}$ are the unknown Fourier coefficients. The depth dependency function $Z_{n}^{3}(z)$ is defined as

$$
Z_{n}^{3}(z)= \begin{cases}1, & \text { for } n=0 \\ \sqrt{2} \cos \left(\alpha_{n}\left(z+e_{1}\right)\right), & \text { for } n \geq 1\end{cases}
$$

with

$$
\alpha_{n}=\frac{n \pi}{e_{1}-b_{1}}
$$


for $n=1,2, \cdots, \infty$.

From the body surface condition (8) we have

$$
\begin{aligned}
& \left.\frac{\partial \varphi_{3}^{3, k}}{\partial z}\right|_{z=-b}=\delta_{1 k}, \text { for } R_{c} \leq r \leq R_{x} \\
& \left.\frac{\partial \varphi_{3}^{3, k}}{\partial z}\right|_{z=-e_{1}}=\delta_{2 k}, \text { for } R_{c} \leq r \leq R_{x}
\end{aligned}
$$

where $R_{x}=\min \left(R_{b}, R_{p}\right)$.

Then the particular solution is given by

$$
\begin{aligned}
\varphi_{3, p}^{3, k}(r, z)= & \frac{1}{2\left(e_{1}-b\right)}\left[\left(z+e_{1}\right)^{2}-\frac{r^{2}}{2}\right] \delta_{1 k} \\
& -\frac{1}{2\left(e_{1}-b\right)}\left[(z+b)^{2}-\frac{r^{2}}{2}\right] \delta_{2 k}
\end{aligned}
$$

Finally for region $\Omega_{4}$ we have

$$
\varphi_{3, h}^{4, k}(r, z)=F_{0}^{k}+\sum_{j=1}^{\infty} F_{j}^{k} \frac{I_{0}\left(\beta_{j} r\right)}{I_{0}\left(\beta_{j} R_{p}\right)} Z_{j}^{4}(z)
$$

where $I_{0}^{(1)}()$ are modified Bessel functions of the first kind and $F_{j}^{k}$ are the unknown Fourier coefficients. The depth dependency function $Z_{j}^{4}(z)$ is defined as

$$
Z_{j}^{4}(z)= \begin{cases}1, & \text { for } j=0 \\ \sqrt{2} \cos \left(\beta_{j}\left(z+e_{2}\right)\right), & \text { for } j \geq 1\end{cases}
$$

with

$$
\beta_{j}=\frac{j \pi}{h-e_{2}}
$$

for $j=1,2, \cdots, \infty$.

From the body surface condition (8) we have

$$
\left.\frac{\partial \varphi_{3}^{4, k}}{\partial z}\right|_{z=-e_{2}}=\delta_{2 k}, \text { for } 0 \leq r \leq R_{p}
$$

and then the particular solution is expressed as

$$
\varphi_{3, p}^{4, k}(r, z)=\frac{1}{2(h-e 2)}\left[(z+h)^{2}-\frac{r^{2}}{2}\right] \delta_{2 k}
$$

All the depth dependency functions $Z_{\tau}^{l}(z)$ are constructed in order to form an orthonormal set of eigenfunctions in the corresponding region $l$.

$$
<Z_{i}^{l}(z), Z_{j}^{l}(z)>=\delta_{i j}
$$

where $\delta_{i j}$ is the Kronecker delta which has been already defined. 


\section{B. Expression of the Scattering Potential in Each Sub-domain}

The scattering potential represents the solution for which the structure is considered fixed in presence of incident waves [18] and is defined as

$$
\phi_{s}(r, \theta, z)=\phi_{0}(r, \theta, z)+\phi_{7}(r, \theta, z)
$$

Considering a linear wave propagating in the positive direction $x$ in water of constant depth $h$ and decribed by a small amplitude $A$ at frequency $\omega$, incident potential $\phi_{0}$ can be defined as

$$
\phi_{0}(r, \theta, z)=B \sum_{m=0}^{\infty} \epsilon^{m} \mathbf{i}^{m} J_{m}\left(k_{e} r\right) Z_{0}^{1}(z) \cos (m \theta)
$$

where $\epsilon^{m}$ is the Neuman symbol defined as $\epsilon^{m}=1$ for $m=0$ and $\epsilon^{m}=2$ otherwise, $J_{m}()$ are Bessel functions of the first kind and $i$ is the complex number. The coefficient $B$ is defined by

$$
B=-\mathrm{i} A \frac{g}{\omega} \frac{1}{Z_{0}^{1}(0)}
$$

Following the same approach as for the radiation problem, we are looking for unknown functions expressed as

$$
\phi_{s}^{l}(r, \theta, z)=\sum_{m=0}^{\infty} \varphi_{s, m}^{l}(r, z) \cos (m \theta)
$$

where only expressions for $m=0$ contribute in the vertical direction. From the gradient condition (8) at all body surfaces we have [1]

$$
\frac{\partial \varphi_{s}}{\partial n}=0
$$

It then follows that the scattering velocity potential in region $\Omega_{1}$ can be expressed as

$$
\begin{aligned}
\varphi_{s, 0}^{1}(r, z)= & B J_{0}\left(k_{e} r\right) Z_{0}^{1}(z)+A_{0}^{0} \frac{H_{0}^{(1)}\left(k_{e} r\right)}{H_{0}^{(1)}\left(k_{e} R_{x}\right)} Z_{0}^{1}(z) \\
& +\sum_{i=1}^{\infty} A_{i}^{0} \frac{K_{0}\left(\lambda_{i} r\right)}{K_{0}\left(\lambda_{i} R_{x}\right)} Z_{i}^{1}(z)
\end{aligned}
$$

where $R_{x}=\max \left(R_{b}, R_{p}\right)$.

One can note that the expression given above has the same form as expression (12) obtained for the radiation problem. For the purpose of the analytical resolution procedure purpose, let us introduce the notation $k=0$, which means that none of the body is moving. Using notations given for the radiation problem we can write

$$
\varphi_{s, 0}^{1}(r, z)=\varphi_{3, h}^{1,0}(r, z)+\varphi_{3, p}^{1,0}(r, z)
$$

where we define

$$
\begin{gathered}
\varphi_{3, h}^{1,0}(r, z)=A_{0}^{0} \frac{H_{0}^{(1)}\left(k_{e} r\right)}{H_{0}^{(1)}\left(k_{e} R_{x}\right)} Z_{0}^{1}(z)+\sum_{i=1}^{\infty} A_{i}^{0} \frac{K_{0}\left(\lambda_{i} r\right)}{K_{0}\left(\lambda_{i} R_{x}\right)} Z_{i}^{1}(z) \\
\varphi_{3, p}^{1,0}(r, z)=B J_{0}\left(k_{e} r\right) Z_{0}^{1}(z)
\end{gathered}
$$

For others regions, unknown functions have the same forms as those developed for the homogeneous part of the radiation problem, that is

$$
\varphi_{s, 0}^{l}(r, z)=\varphi_{3, h}^{l, 0}(r, z)
$$


for $l>1$.

It remains to determine the unknown Fourier coefficients $A_{i}^{k}, B_{l}^{k}, C_{l}^{k}, D_{n}^{k}, E_{n}^{k}$, and $F_{j}^{k}$ in the infinite series of orthogonal functions using the matching eigenfunction expansion method. The coefficients can be determined by applying conditions of pressure and normal velocity continuity at the different imaginary interfaces (i.e. at $r=R_{b}$ and $r=R_{p}$ ) as well as the body surface boundary condition (8) at the body vertical walls.

\section{Solution to the Boundary Value Problem}

\section{A. Determination of the unknown coefficients for $R_{p}=R_{b}$}

In the case where the buoy radius and plate radius are equal, i.e. $R_{p}=R_{b}$, the requirement for pressure and normal velocity continuity has to be fulfilled at the vertical imaginary interface $r=R_{b}$, as well as the boundary conditions on the body vertical surfaces. In heaving mode, the latter are expressed as

$$
\begin{aligned}
& \left.\frac{\partial \varphi_{3}^{1, k}}{\partial r}\right|_{r=R_{p}}=0, \text { for }-e_{2} \leq z \leq-e_{1} \\
& \left.\frac{\partial \varphi_{3}^{1, k}}{\partial r}\right|_{r=R_{b}}=0, \text { for }-b \leq z \leq 0 \\
& \left.\frac{\partial \varphi_{3}^{3, k}}{\partial r}\right|_{r=R_{c}}=0, \text { for }-e_{1} \leq z \leq-b
\end{aligned}
$$

for $k=0,1$ and 2 .

At the imaginary interface $r=R_{b}$, velocity potential continuity conditions are

- for $-e_{1} \leq z \leq-b$

$$
\begin{gathered}
\varphi_{3}^{1, k}\left(R_{b}, z\right)=\varphi_{3}^{3, k}\left(R_{b}, z\right) \\
\left.\frac{\partial \varphi_{3}^{1, k}}{\partial r}\right|_{r=R_{b}}=\left.\frac{\partial \varphi_{3}^{3, k}}{\partial r}\right|_{r=R_{b}}
\end{gathered}
$$

- for $-h \leq z \leq-e_{2}$

$$
\begin{gathered}
\varphi_{3}^{1, k}\left(R_{b}, z\right)=\varphi_{3}^{4, k}\left(R_{b}, z\right) \\
\left.\frac{\partial \varphi_{3}^{1, k}}{\partial r}\right|_{r=R_{b}}=\left.\frac{\partial \varphi_{3}^{4, k}}{\partial r}\right|_{r=R_{b}}
\end{gathered}
$$

Due to the orthogonal properties of the function $Z_{j}^{4}(z)$ valid for $-h \leq z \leq-e_{2}$, it follows that

$$
F_{j}^{k}=\frac{1}{h-e_{2}} \int_{-h}^{-e_{2}} \varphi_{3, h}^{4, k}\left(R_{b}, z\right) Z_{j}^{4}(z) \mathrm{d} z-Q_{j 3}^{k}+O_{j 3}^{k}
$$

where

$$
Q_{j 3}^{k}= \begin{cases}0, & \text { for } k=0 \\ \frac{1}{h-e_{2}} \int_{-h}^{-e_{2}} \varphi_{3, p}^{4, k}\left(R_{b}, z\right) Z_{j}^{4}(z) \mathrm{d} z, & \text { for } k=1,2\end{cases}
$$




$$
O_{j 3}^{k}= \begin{cases}\frac{1}{h-e_{2}} \int_{-h}^{-e_{2}} \varphi_{3, p}^{1,0}\left(R_{b}, z\right) Z_{j}^{4}(z) \mathrm{d} z, & \text { for } k=0 \\ 0, & \text { for } k=1,2\end{cases}
$$

By applying continuity condition (48), Fourier coefficients $F_{j}^{k}$ that describe velocity potential in $\Omega_{4}$ can be expressed in terms of coefficients $A_{i}^{k}$ as follows

$$
F_{j}^{k}=\sum_{i=0}^{\infty} A_{i}^{k} L_{j i}-\left(Q_{j 3}^{k}-O_{j 3}^{k}\right)
$$

with

$$
L_{j i}=\frac{1}{h-e_{2}} \int_{-h}^{-e_{2}} Z_{j}^{4}(z) Z_{i}^{1}(z) \mathrm{d} z
$$

Using the same procedure for interval $-e_{1} \leq z \leq-b$, orthogonal properties of the function $Z_{n}^{3}(z)$ lead to coefficients $D_{n}^{k}$

$$
D_{n}^{k}=\frac{1}{e_{1}-b} \int_{-e_{1}}^{-b} \varphi_{3, h}^{3, k}\left(R_{b}, z\right) Z_{n}^{3}(z) \mathrm{d} z-P_{n 3}^{k}+O_{n 3}^{k}
$$

where

$$
\begin{aligned}
& P_{n 3}^{k}= \begin{cases}0, & \text { for } k=0 \\
\frac{1}{e_{1}-b} \int_{-e_{1}}^{-b} \varphi_{3, p}^{3, k}\left(R_{b}, z\right) Z_{n}^{3}(z) \mathrm{d} z, & \text { for } k=1,2\end{cases} \\
& O_{n 3}^{k}= \begin{cases}\frac{1}{e_{1}-b} \int_{-e_{1}}^{-b} \varphi_{3, p}^{1, k}\left(R_{b}, z\right) Z_{n}^{3}(z) \mathrm{d} z, & \text { for } k=0 \\
0, & \text { for } k=1,2\end{cases}
\end{aligned}
$$

By applying continuity condition (46), Fourier coefficients $D_{n}^{k}$ that describe velocity potential in $\Omega_{3}$ can be expressed in terms of coefficients $A_{i}^{k}$ as follows

$$
D_{n}^{k}=\sum_{i=0}^{\infty} A_{i}^{k} M_{n i}-\left(P_{n 3}^{k}-O_{n 3}^{k}\right)
$$

with

$$
M_{n i}=\frac{1}{e_{1}-b} \int_{-e_{1}}^{-b} Z_{n}^{3}(z) Z_{i}^{1}(z) \mathrm{d} z
$$

By multiplying both sides of equations (43), (44), (47) and (49) by $Z_{\tau}^{1}(z) / h$ (for $\tau=0,1, \cdots, i, \cdots$ ), integrating over the corresponding interval of validity and adding resulting expressions, we obtain a complete set of equations. Replacing coefficients $F_{j}^{k}$ and $D_{n}^{k}$ by their respective definitions (51) and (54) we obtain

$$
h_{\tau}^{k}=\sum_{i=0}^{\infty} d_{\tau i} A_{i}^{k}+\sum_{n=0}^{\infty} d_{\tau n} E_{n}^{k}
$$


with

$$
\begin{aligned}
h_{\tau}^{0}= & B\left(\Upsilon_{0} \delta_{0 \tau}-J_{0}\left(k_{e} R_{b}\right)\left[\frac{h-e_{2}}{h} \sum_{j=0}^{\infty} \Gamma_{j} L_{j 0} L_{j \tau}\right.\right. \\
& \left.\left.+\frac{e_{1}-b}{h} \sum_{n=0}^{\infty} T_{n}^{\prime}\left(R_{b}\right) M_{n 0} M_{n \tau}\right]\right) \\
h_{\tau}^{1}= & \frac{R_{b}}{2 h} M_{0 \tau}+\frac{e_{1}-b}{h} \sum_{n=0}^{\infty} T_{n}^{\prime}\left(R_{b}\right) P_{n 3}^{1} M_{n \tau} \\
h_{\tau}^{2}= & \frac{R_{b}}{2 h}\left(L_{0 \tau}-M_{0 \tau}\right)+\frac{h-e_{2}}{h} \sum_{j=0}^{\infty} \Gamma_{j} Q_{j 3}^{2} L_{j \tau} \\
& +\frac{e_{1}-b}{h} \sum_{n=0}^{\infty} T_{n}^{\prime}\left(R_{b}\right) P_{n 3}^{2} M_{n \tau} \\
d_{\tau i}= & \Delta_{i} \delta_{i \tau}+\frac{h-e_{2}}{h} \sum_{j=0}^{\infty} \Gamma_{j} L_{j i} L_{j \tau} \\
& +\frac{e_{1}-b}{h} \sum_{n=0}^{\infty} T_{n}^{\prime}\left(R_{b}\right) M_{n i} M_{n \tau} \\
d_{\tau n}= & \frac{e_{1}-b}{h} \sum_{n=0}^{\infty} \tilde{T}_{n}^{\prime}\left(R_{b}\right) M_{n \tau}
\end{aligned}
$$

where we define

$$
\begin{aligned}
& \Gamma_{0}=0 \\
& \Gamma_{j}=\beta_{j} \frac{I_{1}\left(\beta_{j} R_{b}\right)}{I_{0}\left(\beta_{j} R_{b}\right)} \\
& \Delta_{0}=k_{e} \frac{H_{1}^{(1)}\left(k_{e} R_{b}\right)}{H_{0}^{(1)}\left(k_{e} R_{b}\right)} \quad \Delta_{i}=\lambda_{i} \frac{K_{1}\left(\lambda_{i} R_{b}\right)}{K_{0}\left(\lambda_{i} R_{b}\right)}
\end{aligned}
$$

for $i, j \geq 1$ and

$$
\Upsilon_{0}=-k_{e} J_{1}\left(k_{e} R_{b}\right)
$$

Finally, multiplying the body surface condition (45), expressed on the column, by $Z_{\nu}^{3}(z) /\left(e_{1}-b\right)$ (for $\nu=$ $0,1, \cdots, n, \cdots)$, integrating with respect to $z$, i.e. $-e_{1} \leq z \leq-b$, and using the definition of $D_{n}^{k}$, we obtain the last set of equations at the vertical boundary $r=R_{c}$.

$$
h_{\nu}^{k}=\sum_{i=0}^{\infty} d_{\nu i} A_{i}^{k}+\sum_{n=0}^{\infty} d_{\nu n} E_{n}^{k}
$$

where

$$
\begin{aligned}
h_{\nu}^{0} & =-B J_{0}\left(k_{e} R_{c}\right) \sum_{n=0}^{\infty} T_{n}^{\prime}\left(R_{c}\right) M_{n 0} \delta_{n \nu} \\
h_{\nu}^{1} & =\sum_{n=0}^{\infty} T_{n}^{\prime}\left(R_{c}\right) P_{n 3}^{1} \delta_{n \nu}+\frac{R_{c}}{2\left(e_{1}-b\right)} \delta_{0 \nu} \\
h_{\nu}^{2} & =\sum_{n=0}^{\infty} T_{n}^{\prime}\left(R_{c}\right) P_{n 3}^{2} \delta_{n \nu}-\frac{R_{c}}{2\left(e_{1}-b\right)} \delta_{0 \nu}
\end{aligned}
$$




$$
\begin{aligned}
d_{\nu i} & =\sum_{n=0}^{\infty} T_{n}^{\prime}\left(R_{c}\right) M_{n i} \delta_{n \nu} \\
d_{\nu n} & =\tilde{T}_{n}^{\prime}\left(R_{c}\right) \delta_{n \nu}
\end{aligned}
$$

In order to find a solution to unknown Fourier coefficients $A_{i}^{k}$ and $E_{n}^{k}$, we have to truncate the infinite series to the first $N$ terms. By introducing matrix notation, the unknown coefficients can be determined, solving simultaneously the two sets of equations (56) and (65) which can be expressed by the following linear system

$$
\left[\begin{array}{ll}
d_{\tau i} & d_{\tau n} \\
d_{\nu i} & d_{\nu n}
\end{array}\right]\left(\begin{array}{c}
A_{i 1}^{k} \\
E_{n 1}^{k}
\end{array}\right)=\left[\begin{array}{c}
h_{\tau 1}^{k} \\
\\
h_{\nu 1}^{k}
\end{array}\right]
$$

Note that the matrix on the left hand side of (71) does not depend on the boundary value conditions of the BVP. The matrix term values would only change if we modify the geometrical configuration. This can be an advantage in terms of reducing the numerical computation time. Finally, the remaining Fourier coefficients $F_{j}^{k}$ and $D_{n}^{k}$ can be computed respectively from equations (51) and (54).

\section{B. Determination of the unknown coefficients for $R_{p}>R_{b}$}

In the case where the plate radius is greater than the buoy radius, i.e. $R_{p}>R_{b}$, the requirement for pressure and normal velocity continuity has to be fulfilled at the two vertical imaginary interfaces $r=R_{p}$ and $r=R_{b}$, as well as the boundary value conditions on the body vertical surfaces. In heaving mode the latter are expressed as

$$
\begin{aligned}
& \left.\frac{\partial \varphi_{3}^{1, k}}{\partial r}\right|_{r=R_{p}}=0, \text { for }-e_{2} \leq z \leq-e_{1} \\
& \left.\frac{\partial \varphi_{3}^{2, k}}{\partial r}\right|_{r=R_{b}}=0, \text { for }-b \leq z \leq 0 \\
& \left.\frac{\partial \varphi_{3}^{3, k}}{\partial r}\right|_{r=R_{c}}=0, \text { for }-e_{1} \leq z \leq-b
\end{aligned}
$$

Specifying velocity potential continuity conditions at the imaginary interface $r=R_{p}$ we have

- for $-h \leq z \leq-e_{2}$

$$
\begin{gathered}
\varphi_{3}^{1, k}\left(R_{p}, z\right)=\varphi_{3}^{4, k}\left(R_{p}, z\right) \\
\left.\frac{\partial \varphi_{3}^{1, k}}{\partial r}\right|_{r=R_{p}}=\left.\frac{\partial \varphi_{3}^{4, k}}{\partial r}\right|_{r=R_{p}}
\end{gathered}
$$

- for $-e_{1} \leq z \leq 0$ 


$$
\begin{gathered}
\varphi_{3}^{1, k}\left(R_{p}, z\right)=\varphi_{3}^{2, k}\left(R_{p}, z\right) \\
\left.\frac{\partial \varphi_{3}^{1, k}}{\partial r}\right|_{r=R_{p}}=\left.\frac{\partial \varphi_{3}^{2, k}}{\partial r}\right|_{r=R_{p}}
\end{gathered}
$$

Using the orthogonal properties of the trigonometric function $Z_{j}^{4}(z)$ valid for $-h \leq z \leq-e_{2}$, it follows that for $r=R_{p}$

$$
F_{j}^{k}=\frac{1}{h-e_{2}} \int_{-h}^{-e_{2}} \varphi_{3, p}^{4, k}\left(R_{p}, z\right) Z_{j}^{4}(z) \mathrm{d} z-Q_{j 3}^{k}+O_{j 3}^{k}
$$

where

$$
\begin{aligned}
& Q_{j 3}^{k}= \begin{cases}0, & \text { for } k=0 \\
\frac{1}{h-e_{2}} \int_{-h}^{-e_{2}} \varphi_{3, p}^{4, k}\left(R_{p}, z\right) Z_{j}^{4}(z) \mathrm{d} z, & \text { for } k=1,2\end{cases} \\
& O_{j 3}^{k}= \begin{cases}\frac{1}{h-e_{2}} \int_{-h}^{-e_{2}} \varphi_{3, p}^{1,0}\left(R_{p}, z\right) Z_{j}^{4}(z) \mathrm{d} z, & \text { for } k=0 \\
0, & \text { for } k=1,2\end{cases}
\end{aligned}
$$

From the normal velocity continuity condition (75) we can expressed $F_{j}^{k}$ as

$$
F_{j}^{k}=\sum_{i=0}^{\infty} A_{i}^{k} L_{j i}-\left(Q_{j 3}^{k}-O_{j 3}^{k}\right)
$$

with

$$
L_{j i}=\frac{1}{h-e_{2}} \int_{-h}^{-e_{2}} Z_{j}^{4}(z) Z_{i}^{1}(z) \mathrm{d} z
$$

In a similar way, by using orthogonal characteristics of the set $Z_{l}^{2}(z)$ valid in $-e_{1} \leq z \leq 0$ and from (77), we can express $B_{l}^{k}$ as

$$
B_{l}^{k}=\sum_{i=0}^{\infty} K_{l i} A_{i}^{k}-\left(R_{l 3}^{k}-O_{l 3}^{k}\right)
$$

with

$$
K_{l i}=\frac{1}{e_{1}} \int_{-e_{1}}^{0} Z_{l}^{2}(z) Z_{i}^{1}(z) \mathrm{d} z
$$

and

$$
\begin{aligned}
& R_{l 3}^{k}= \begin{cases}0, & \text { for } k=0 \\
\frac{1}{e_{1}} \int_{-e_{1}}^{0} \varphi_{3, p}^{2, k}\left(R_{p}, z\right) Z_{l}^{2}(z) \mathrm{d} z, & \text { for } k=1,2\end{cases} \\
& O_{l 3}^{k}= \begin{cases}\frac{1}{e_{1}} \int_{-e_{1}}^{0} \varphi_{3, p}^{1,0}\left(R_{p}, z\right) Z_{l}^{2}(z) \mathrm{d} z, & \text { for } k=0 \\
0, & \text { for } k=1,2\end{cases}
\end{aligned}
$$

By multiplying both sides of equations (76), (72) and (78) by $Z_{\tau}^{1}(z) / h$ (for $\tau=0,1, \cdots, i, \cdots$ ), integrating over the corresponding domain of validity and adding resulting expressions, a complete set of equations is formed. Replacing coefficients $F_{j}^{k}$ and $B_{l}^{k}$ by their respective definitions (80) and (82), we obtain

$$
h_{\tau}^{k}=\sum_{i=0}^{\infty} d_{\tau i} A_{i}^{k}+\sum_{l=0}^{\infty} d_{\tau l} C_{l}^{k}
$$


where

$$
\begin{aligned}
h_{\tau}^{0}= & B\left(\Upsilon_{0} \delta_{0 \tau}-J_{0}\left(k_{e} R_{p}\right)\left[\frac{h-e_{2}}{h} \sum_{j=0}^{\infty} \Gamma_{j} L_{j 0} L_{j \tau}\right.\right. \\
& \left.\left.+\frac{e_{1}}{h} \sum_{l=0}^{\infty} S_{l}^{\prime}\left(R_{p}\right) K_{l 0} K_{l \tau}\right]\right) \\
h_{\tau}^{1}= & 0 \\
h_{\tau}^{2}= & \frac{R_{p}}{2 h} L_{0 \tau}+\frac{h-e_{2}}{h} \sum_{j=0}^{\infty} \Gamma_{j} Q_{j 3}^{2} L_{j \tau} \\
& +\frac{e_{1}}{h} \sum_{l=0}^{\infty} S_{l}^{\prime}\left(R_{p}\right) R_{l 3}^{2} K_{l \tau} \\
d_{\tau i}= & \Delta_{i} \delta_{i \tau}+\frac{h-e_{2}}{h} \sum_{j=0}^{\infty} \Gamma_{j} L_{j i} L_{j \tau}+\frac{e_{1}}{h} \sum_{l=0}^{\infty} S_{l}^{\prime}\left(R_{p}\right) K_{l i} K_{l \tau} \\
d_{\tau l}= & \frac{e_{1}}{h}\left[\tilde{S}_{l}^{\prime}\left(R_{p}\right) K_{l \tau}\right]
\end{aligned}
$$

The same procedure as the one used above is applied at the imaginary interfaces $r=R_{b}$. From the normal velocity continuity condition (90)

$$
\varphi_{3}^{2, k}\left(R_{b}, z\right)=\varphi_{3}^{3, k}\left(R_{b}, z\right)
$$

the Fourier coefficients $D_{n}^{k}$ can be expressed as

$$
D_{n}^{k}=\sum_{l=0}^{\infty} M_{n l} C_{l}^{k}-\left(P_{n 3}^{k}-R_{n 3}^{k}\right)
$$

where

$$
M_{n l}=\frac{1}{e_{1}-b} \int_{-e_{1}}^{-b} Z_{n}^{3}(z) Z_{l}^{2}(z) \mathrm{d} z
$$

and

$$
\begin{aligned}
P_{n 3}^{k} & =\frac{1}{e_{1}-b} \int_{-e_{1}}^{-b} \varphi_{3, p}^{3, k}\left(R_{b}, z\right) Z_{n}^{3}(z) \mathrm{d} z \\
R_{n 3}^{k} & =\frac{1}{e_{1}-b} \int_{-e_{1}}^{-b} \varphi_{3, p}^{2, k}\left(R_{b}, z\right) Z_{n}^{3}(z) \mathrm{d} z
\end{aligned}
$$

for $k=1,2$ and 0 otherwise.

The pressure continuity condition

$$
\left.\frac{\partial \varphi_{3}^{2, k}}{\partial r}\right|_{r=R_{b}}=\left.\frac{\partial \varphi_{3}^{3, k}}{\partial r}\right|_{r=R_{b}}
$$

as well as the body surface boundary condition (73) on the buoy have to be fulfilled. Multiplying both sides of these two conditions by $Z_{\nu}^{2}(z) / e_{1}$ (for $\nu=0,1, \cdots, l, \cdots$ ), integrating over the corresponding interval and adding resulting expressions, a complete set of equations is formed.

$$
h_{\nu}^{k}=\sum_{i=0}^{\infty} d_{\nu i} A_{i}^{k}+\sum_{l=0}^{\infty} d_{\nu l} C_{l}^{k}+\sum_{n=0}^{\infty} d_{\nu n} E_{n}^{k}
$$


where

$$
\begin{aligned}
h_{\nu}^{0}= & B J_{0}\left(k_{e} R_{p}\right) \sum_{l=0}^{\infty} S_{l}^{\prime}\left(R_{b}\right) K_{l 0} \delta_{l \nu} \\
h_{\nu}^{1}= & \sum_{n=0}^{\infty} T_{n}^{\prime}\left(R_{b}\right) P_{n 3}^{1} \delta_{n \nu}+\frac{R_{b}}{2 e_{1}} M_{0 \nu} \\
h_{\nu}^{2}= & -\sum_{l=0}^{\infty} S_{l}^{\prime}\left(R_{b}\right) R_{l 3}^{2} \delta_{l \nu} \\
& +\sum_{n=0}^{\infty} T_{n}^{\prime}\left(R_{b}\right)\left(P_{n 3}^{2}-S_{n 3}^{2}\right) \delta_{n \nu}-\frac{R_{b}}{2 e_{1}} M_{0 \nu} \\
d_{\nu i}= & -\sum_{l=0}^{\infty} S_{l}^{\prime}\left(R_{b}\right) K_{l i} \delta_{l \nu} \\
d_{\nu l}= & -S_{l}^{\prime}\left(R_{b}\right) \delta_{l \nu}+\frac{e_{1}-b}{e_{1}} \sum_{n=0}^{\infty} T_{n}^{\prime}\left(R_{b}\right) M_{n l} M_{n \nu} \\
d_{\nu n}= & \frac{e_{1}-b}{e_{1}}\left[\tilde{T}_{n}^{\prime}\left(R_{b}\right) M_{n \nu}\right]
\end{aligned}
$$

Finally, multiplying the body surface condition (74), expressed on the column, by $Z_{\sigma}^{3}(z) /\left(e_{1}-b\right)$ (for $\sigma=0,1, \cdots, n, \cdots$ ) and integrating with respect to $z$, i.e. $-e_{1} \leq z \leq-b$, the last set of equations at the imaginary interface $r=R_{c}$ is obtained

$$
h_{\sigma}^{k}=\sum_{l=0}^{\infty} d_{\sigma l} C_{l}^{k}+\sum_{n=0}^{\infty} d_{\sigma n} E_{n}^{k}
$$

where

$$
\begin{aligned}
h_{\sigma}^{0} & =0 \\
h_{\sigma}^{1} & =\sum_{n=0}^{\infty} T_{n}^{\prime}\left(R_{c}\right) P_{n 3}^{1} \delta_{n \sigma}+\frac{R_{c}}{2\left(e_{1}-b\right)} \delta_{0 \sigma} \\
h_{\sigma}^{2} & =\sum_{n=0}^{\infty} T_{n}^{\prime}\left(R_{c}\right) P_{n 3}^{2} \delta_{n \sigma}-\frac{R_{c}}{2\left(e_{1}-b\right)} \delta_{0 \sigma} \\
d_{\sigma l} & =\sum_{n=0}^{\infty} T_{n}^{\prime}\left(R_{c}\right) M_{n l} \delta_{n \sigma} \\
d_{\sigma n} & =\tilde{T}_{n}^{\prime}\left(R_{c}\right) \delta_{n \sigma}
\end{aligned}
$$

In order to find a solution to unknown Fourier coefficients $A_{i}^{k}, C_{l}^{k}$, and $E_{n}^{k}$ we have to truncate the infinite series to the first $N$ terms. The unknown coefficients can be determined by solving the linear system (109) and the remaining coefficients $B_{l}^{k}, D_{n}^{k}$, and $F_{j}^{k}$ can be obtained respectively from their expressions (82), (91), and (80).

$$
\left[\begin{array}{ccc}
d_{\tau i} & d_{\tau l} & 0 \\
d_{\nu i} & d_{\nu l} & d_{\nu n} \\
0 & d_{\sigma l} & d_{\sigma n}
\end{array}\right]\left(\begin{array}{c}
A_{i 1}^{k} \\
C_{l 1}^{k} \\
E_{n 1}^{k}
\end{array}\right)=\left[\begin{array}{c}
h_{\tau 1}^{k} \\
h_{\nu 1}^{k} \\
h_{\sigma 1}^{k}
\end{array}\right]
$$


C. Determination of the unknown coefficients for $R_{p}<R_{b}$

In the case where the plate radius is smaller than the buoy radius, i.e. $R_{p}<R_{b}$, the requirement for pressure and normal velocity continuity has to be fulfilled at the two vertical imaginary interfaces $r=R_{p}$ and $r=R_{b}$, as well as the boundary conditions on the body vertical surfaces. In heaving mode the latter are expressed as

$$
\begin{aligned}
& \left.\frac{\partial \varphi_{3}^{1, k}}{\partial r}\right|_{r=R_{b}}=0, \text { for }-b \leq z \leq 0 \\
& \left.\frac{\partial \varphi_{3}^{2, k}}{\partial r}\right|_{r=R_{p}}=0, \text { for }-e_{2} \leq z \leq-e_{1} \\
& \left.\frac{\partial \varphi_{3}^{3, k}}{\partial r}\right|_{r=R_{c}}=0, \text { for }-e_{1} \leq z \leq-b
\end{aligned}
$$

Specifying the normal velocity continuity condition at the imaginary interface $r=R_{b}$, we have

$$
\varphi_{3}^{1, k}\left(R_{b}, z\right)=\varphi_{3}^{2, k}\left(R_{b}, z\right)
$$

for $-h \leq z \leq-b$ and unknown Fourier coefficients $B_{l}^{k}$ that describe velocity potential in sub-domain $\Omega_{2}$ can then be expressed as

$$
B_{l}^{k}=\sum_{i=0}^{\infty} K_{l i} A_{i}^{k}-\left(R_{l 3}^{k}-O_{l 3}^{k}\right)
$$

where

$$
K_{l i}=\frac{1}{h-b} \int_{-h}^{-b} Z_{l}^{2}(z) Z_{i}^{1}(z) \mathrm{d} z
$$

and

$$
\begin{aligned}
& R_{l 3}^{k}= \begin{cases}0, & \text { for } k=0 \\
\frac{1}{h-b} \int_{-h}^{-b} \varphi_{3, p}^{2, k}\left(R_{b}, z\right) Z_{l}^{2}(z) \mathrm{d} z, & \text { for } k=1,2\end{cases} \\
& O_{l 3}^{k}= \begin{cases}\frac{1}{h-b} \int_{-h}^{-b} \varphi_{3, p}^{1,0}\left(R_{b}, z\right) Z_{l}^{2}(z) \mathrm{d} z, & \text { for } k=0 \\
0, & \text { for } k=1,2\end{cases}
\end{aligned}
$$

From gradient condition (110) and applying the pressure continuity condition

$$
\left.\frac{\partial \varphi_{3}^{1, k}}{\partial r}\right|_{r=R_{b}}=\left.\frac{\partial \varphi_{3}^{2, k}}{\partial r}\right|_{r=R_{b}}
$$

a complete set of equations is formed by multiplying both sides of these last conditions by $Z_{\tau}^{1}(z) / h$ (for $\tau=0,1, \cdots, i, \cdots$ ); following a similar procedure as described above. Using expressions (114) for coefficients $B_{l}^{k}$, we obtain

$$
h_{\tau}^{k}=\sum_{i=0}^{\infty} d_{\tau i} A_{i}^{k}+\sum_{l=0}^{\infty} d_{\tau l} C_{l}^{k}
$$


for which we define

$$
\begin{aligned}
h_{\tau}^{0}= & B\left[\Upsilon_{0} \delta_{0 \tau}-J_{0}\left(k_{e} R_{b}\right) \frac{h-b}{h} \sum_{l=0}^{\infty} S_{l}^{\prime}\left(R_{b}\right) K_{l 0} K_{l \tau}\right] \\
h_{\tau}^{1}= & \frac{R_{b}}{2 h} K_{0 \tau}+\frac{h-b}{h} \sum_{l=0}^{\infty} S_{l}^{\prime}\left(R_{b}\right) R_{l 3}^{1} K_{l \tau} \\
h_{\tau}^{2}= & 0 \\
d_{\tau i}= & \Delta_{i} \delta_{i \tau}+\frac{h-b}{h} \sum_{l=0}^{\infty} S_{l}^{\prime}\left(R_{b}\right) K_{l i} K_{l \tau} \\
d_{\tau l}= & \frac{h-b}{h}\left[\tilde{S}_{l}^{\prime}\left(R_{b}\right) K_{l \tau}\right]
\end{aligned}
$$

At the imaginary interface $r=R_{p}$, for continuity conditions we have

- for $-h \leq z \leq-e_{2}$

$$
\begin{gathered}
\varphi_{3}^{2, k}\left(R_{p}, z\right)=\varphi_{3}^{4, k}\left(R_{p}, z\right) \\
\left.\frac{\partial \varphi_{3}^{2, k}}{\partial r}\right|_{r=R_{p}}=\left.\frac{\partial \varphi_{3}^{4, k}}{\partial r}\right|_{r=R_{p}}
\end{gathered}
$$

- for $-e_{1} \leq z \leq-b$

$$
\begin{aligned}
& \varphi_{3}^{2, k}\left(R_{p}, z\right)=\varphi_{3}^{3, k}\left(R_{p}, z\right) \\
& \left.\frac{\partial \varphi_{3}^{2, k}}{\partial r}\right|_{r=R_{p}}=\left.\frac{\partial \varphi_{3}^{3, k}}{\partial r}\right|_{r=R_{p}}
\end{aligned}
$$

From condition (123) we can express $F_{j}^{k}$ as

$$
F_{j}^{k}=\sum_{l=0}^{\infty} C_{l}^{k} L_{j l}-\left(Q_{j 3}^{k}-R_{j 3}^{k}\right)
$$

where

$$
L_{j l}=\frac{1}{h-e_{2}} \int_{-h}^{-e_{2}} Z_{j}^{4}(z) Z_{l}^{2}(z) \mathrm{d} z
$$

and

$$
\begin{aligned}
Q_{j 3}^{k} & =\frac{1}{h-e_{2}} \int_{-h}^{-e_{2}} \varphi_{3, p}^{4, k}\left(R_{p}, z\right) Z_{j}^{4}(z) \mathrm{d} z \\
R_{j 3}^{k} & =\frac{1}{h-e_{2}} \int_{-h}^{-e_{2}} \varphi_{3, p}^{2, k}\left(R_{p}, z\right) Z_{j}^{4}(z) \mathrm{d} z
\end{aligned}
$$

for $k=1,2$ and 0 otherwise.

From condition (125) we can express $D_{n}^{k}$ as

$$
D_{n}^{k}=\sum_{l=0}^{\infty} M_{n l} C_{l}^{k}-\left(P_{n 3}^{k}-R_{n 3}^{k}\right)
$$


with

$$
M_{n l}=\frac{1}{e_{1}-b} \int_{-e_{1}}^{-b} Z_{n}^{3}(z) Z_{l}^{2}(z) \mathrm{d} z
$$

and

$$
\begin{aligned}
& P_{n 3}^{k}=\frac{1}{e_{1}-b} \int_{-e_{1}}^{-b} \varphi_{3, p}^{3, k}\left(R_{p}, z\right) Z_{n}^{3}(z) \mathrm{d} z \\
& R_{n 3}^{k}=\frac{1}{e_{1}-b} \int_{-e_{1}}^{-b} \varphi_{3, p}^{2, k}\left(R_{p}, z\right) Z_{n}^{3}(z) \mathrm{d} z
\end{aligned}
$$

By multiplying both sides of equations (124), (111), and (126) by $Z_{\nu}^{1}(z) /(h-b)$ (for $\left.\nu=0,1, \cdots, l, \cdots\right)$, integrating over the corresponding interval of validity and adding resulting expressions, a complete set of equations is obtained. Replacing coefficients $B_{l}^{k}, F_{j}^{k}$, and $D_{n}^{k}$ by their respective definitions (114), (127), and (131) we obtain

$$
h_{\nu}^{k}=\sum_{i=0}^{\infty} d_{\nu i} A_{i}^{k}+\sum_{l=0}^{\infty} d_{\nu l} C_{l}^{k}+\sum_{n=0}^{\infty} d_{\nu n} E_{n}^{k}
$$

where

$$
\begin{aligned}
h_{\nu}^{0}= & B J_{0}\left(k_{e} R_{b}\right) \sum_{l=0}^{\infty} S_{l}^{\prime}\left(R_{p}\right) K_{l 0} \delta_{l \nu} \\
h_{\nu}^{1}= & \frac{R_{p}}{2(h-b)}\left(M_{0 \nu}-\delta_{0 \nu}\right)-\sum_{l=0}^{\infty} S_{l}^{\prime}\left(R_{p}\right) R_{l 3}^{1} \delta_{l \nu} \\
& +\frac{e_{1}-b}{h-b} \sum_{n=0}^{\infty} T_{n}^{\prime}\left(R_{p}\right)\left(P_{n 3}^{1}-R_{n 3}^{1}\right) M_{n \nu} \\
& -\frac{h-e_{2}}{h-b} \sum_{j=0}^{\infty} \Gamma_{j} R_{j 3}^{1} L_{j \nu} \\
h_{\nu}^{2}= & \frac{R_{p}}{2(h-b)}\left(L_{0 \nu}-M_{0 \nu}\right)+\frac{h-e_{2}}{h-b} \sum_{j=0}^{\infty} \Gamma_{j} Q_{j 3}^{2} L_{j \nu} \\
& +\frac{e_{1}-b}{h-b} \sum_{n=0}^{\infty} T_{n}^{\prime}\left(R_{p}\right) P_{n 3}^{2} M_{n \nu} \\
d_{\nu i}= & -\sum_{l=0}^{\infty} S_{l}^{\prime}\left(R_{p}\right) K_{l i} \delta_{l \nu} \\
d_{\nu l}= & -\tilde{S}_{l}^{\prime}\left(R_{p}\right) \delta_{l \nu}+\frac{h-e_{2}}{h-b} \sum_{j=0}^{\infty} \Gamma_{j} L_{j l} L_{j \nu} \\
& +\frac{e_{1}-b}{h-b} \sum_{n=0}^{\infty} T_{n}^{\prime}\left(R_{p}\right) M_{n l} M_{n \nu} \\
d_{\nu n}= & \frac{e_{1}-b}{h-b}\left[\tilde{T}_{n}^{\prime}\left(R_{p}\right) M_{n \nu}\right]
\end{aligned}
$$

Finally, multiplying the body surface condition (112), expressed on the column, by $Z_{\sigma}^{3}(z) /\left(e_{1}-b\right)$ (for $\sigma=$ $0,1, \cdots, n, \cdots)$ and integrating with respect to $z$, i.e. $-e_{1} \leq z \leq-b$, we obtain the last set of equations at the imaginary interface $r=R_{c}$.

$$
h_{\sigma}^{k}=\sum_{l=0}^{\infty} d_{\sigma l} C_{l}^{k}+\sum_{n=0}^{\infty} d_{\sigma n} E_{n}^{k}
$$


where

$$
\begin{aligned}
h_{\sigma}^{0} & =0 \\
h_{\sigma}^{1} & =\sum_{n=0}^{\infty} T_{n}^{\prime}\left(R_{c}\right)\left(P_{n 3}^{1}-S_{n 3}^{1}\right) \delta_{n \sigma}+\frac{R_{c}}{2\left(e_{1}-b\right)} \delta_{0 \sigma} \\
h_{\sigma}^{2} & =\sum_{n=0}^{\infty} T_{n}^{\prime}\left(R_{c}\right) P_{n 3}^{2} \delta_{n \sigma}-\frac{R_{c}}{2\left(e_{1}-b\right)} \delta_{0 \sigma} \\
d_{\sigma l} & =\sum_{n=0}^{\infty} T_{n}^{\prime}\left(R_{c}\right) M_{n l} \delta_{n \sigma} \\
d_{\sigma n} & =\tilde{T}_{n}^{\prime}\left(R_{c}\right) \delta_{n \sigma}
\end{aligned}
$$

Using matrix notation, the unknown Fourier coefficients $A_{i}^{k}, C_{l}^{k}$, and $E_{n}^{k}$ can be determined by solving the following linear system.

$$
\left[\begin{array}{ccc}
d_{\tau i} & d_{\tau l} & 0 \\
d_{\nu i} & d_{\nu l} & d_{\nu n} \\
0 & d_{\sigma l} & d_{\sigma n}
\end{array}\right]\left(\begin{array}{c}
A_{i 1}^{k} \\
C_{l 1}^{k} \\
E_{n 1}^{k}
\end{array}\right)=\left[\begin{array}{c}
h_{\tau 1}^{k} \\
h_{\nu 1}^{k} \\
h_{\sigma 1}^{k}
\end{array}\right]
$$

The remaining coefficients $B_{l}^{k}, D_{n}^{k}$, and $F_{j}^{k}$ can be obtained respectively from their expressions (114), (131), and (127).

\section{EXCITATION Forces AND HydrodynamiC COEFFICIENTS}

Once the scattering and/or radiation problems are solved, which means that we determined the unknown Fourier coefficients for the orthogonal series, the velocity potential is known in the whole fluid domain. Waves exciting forces and/or hydrodynamic coefficients can then be determined by integrating the hydrodynamic pressure, given by the Bernoulli equation, over the wet surface of the body under consideration. Based on the linear wave theory, the pressure is given by

$$
p(r, \theta, z, t)=-\rho \frac{\partial \Phi}{\partial t}=\mathrm{i} \omega \rho \phi(r, \theta, z) e^{-\mathrm{i} \omega t}
$$

\section{A. Added Mass and Radiation Damping Coefficients}

When the structure is moving, a radiation force acts on it and can be expressed as

$$
F_{q, j}^{k, i}(\omega)=\mathrm{i} \omega \rho \iint_{S_{i}} \phi_{q}^{k}(r, \theta, z) n_{j} \mathrm{~d} S
$$

where $F_{q, j}^{k, i}(\omega)$ is the complex representation of the radiation force acting on the body $i$ in the direction $j$ due to the motion of the body $k$ in the direction $q$ (here $q=3$ ). It is conventional to break down this radiation force into two components: one proportional to the acceleration of body and the other proportional to his velocity as follows

$$
F_{q, j}^{k, i}(\omega)=-\omega^{2} \zeta_{q}\left[\mu_{q, j}^{k, i}+\frac{\mathrm{i}}{\omega} \lambda_{q, j}^{k, i}\right]
$$


where hydrodynamic coefficients $\mu_{q, j}^{k, i}$ and $\lambda_{q, j}^{k, i}$ are referenced in the literature as the added mass and the radiation or potential damping, respectively. Finally, using expression (11) in heaving mode we obtain

$$
\mu_{3,3}^{k, i}+\frac{\mathrm{i}}{\omega} \lambda_{3,3}^{k, i}=2 \pi \rho \sum_{l=1}^{L}\left[\int_{S_{i}} \phi_{3}^{l, k}(r, z) n_{3} r \mathrm{~d} r\right]
$$

\section{B. Wave Excitation Forces in z Direction}

When the structure is considered as fixed in the presence of an incident wave, an excitation force acts on the bodies. Using a complex representation, excitation forces in the vertical direction can be expressed in terms of scattering potential, see (35), as

$$
f_{z}^{k}(\omega)=\mathrm{i} \omega \rho \iint_{S_{k}} \phi_{s}(r, \theta, z) n_{3} \mathrm{~d} S
$$

where $k$ indicates which body is under consideration and $n_{3}$ is the component of the generalised normal vector in $z$ direction. The Haskind's relation is another approach for evaluating wave excitation forces [20], [21], [19]. They can be evaluated either at the wet body surface $S_{k}$ by (154)

$$
f_{z}^{k}(\omega)=\mathrm{i} \omega \rho \iint_{S_{k}}\left(\phi_{0} \frac{\partial \phi_{3}}{\partial n}-\phi_{3} \frac{\partial \phi_{0}}{\partial n}\right) \mathrm{d} S
$$

or at the control surface $S_{\infty}$ by (155).

$$
f_{z}^{k}(\omega)=-\mathrm{i} \omega \rho \lim _{r \rightarrow \infty} \int_{0}^{2 \pi} \int_{-h}^{0}\left[\phi_{0} \frac{\partial \varphi_{3,0}^{1, k}}{\partial r}-\varphi_{3,0}^{1, k} \frac{\partial \phi_{0}}{\partial r}\right] r \mathrm{~d} z \mathrm{~d} \theta
$$

where we take, as control surface $S_{\infty}$, a vertical cylinder about the $z$ axis of large radius $r$, as recommended in [21].

The main advantage of these two relations is that we only need to know solutions for the incident and radiated potential. However, as already mentioned in [12], evaluating expressions obtained by (154) is complicated since potentials have to be integrated on the whole of the wet structure. Therefore, the use of the far-field behaviour of the radiation solution given by (155), which simplifies the analysis is used here. After integral evaluation, we find that the wave excitation force for the heaving mode is (see [15] for details)

$$
f_{z}^{k}(\omega)=\frac{-4 \mathrm{i} \rho g h \sqrt{N_{\lambda_{0}}} A_{0}^{k}}{\cosh \left(k_{e} h\right) H_{0}^{(1)}\left(k_{e} R_{x}\right)}
$$

\section{Numerical Results AND Discussion}

A specific code based on the above formulation has been developed in order to solve problems (71), (109) and (148). To carry out numerical computations, the infinite series in the expressions of the radiated and scattering potentials have to be truncated to a finite number of terms. According to the literature [4], [5], [12], it seems that considering only the first 30 terms shows good truncation characteristics. We have therefore chosen $N=30$ for potential expressions in all sub-domains.

In order to validate the analytical expressions obtained for velocity potentials, several checks are performed on numerical results and then different case studies have to be considered. In Table I, we have summarised 
geometrical configurations for each case study where $\alpha$ and $\beta$ are two variable parameters defined as $\alpha \in$ $\left[\begin{array}{llllll}8 & 2 & 1 & .75 & .65 & .51\end{array}\right]$ and $\beta \in\left[\begin{array}{llll}.5 & .35 & .25 & .15\end{array}\right]$. They represent respectively the submerged plate to buoy radius ratio and the column to buoy radius ratio and they are used to cover all the test scenarios. Geometrical parameters for case studies no.1 and no.3 are taken respectively from models developed by Wu et al. [14] and Chau and Yeung [15]. For all numerical computations, we have used $h=1$. For the scattering problem, solutions have been obtained based on the assumption of a unit amplitude incident wave, $A=1$.

TABLE I

GEOMETRICAL PARAMETERS FOR NUMERICAL COMPUTATION

\begin{tabular}{l|cccccc}
\hline Case No. & $b / h$ & $R_{b} / h$ & $e_{1} / h$ & $e_{2} / h$ & $R_{p} / R_{b}$ & $R_{c} / R_{b}$ \\
\hline \hline 1 & 0.1 & 0.2 & 0.25 & 0.35 & $\alpha$ & $10^{-3}$ \\
2 & 0.1 & 0.2 & 0.25 & 0.35 & $\alpha$ & 0.5 \\
3 & 0.25 & 1 & 0.4 & 0.5 & $\alpha$ & 0.5 \\
4 & 0.25 & 1 & 0.4 & 0.5 & $\alpha$ & $\beta$ \\
\hline
\end{tabular}

\section{A. Vertical boundary condition matching}

We first look at the matching of the velocity potential and its first derivative in the radial direction along the imaginary interfaces between sub-domains. In Fig. 2, results are presented for the structure depicted in Section I for the case study No.3, where the plate radius is smaller than that of the buoy, $\alpha=.75$, and where the buoy is moving and the platform is fixed. Excellent matching is achieved on velocity potential using the above numerical truncation. However, for gradient visualisation purposes, we have used more terms in expressions to reduce the oscillations due to Bessel functions ( $N=80$ for regions $\Omega_{1}, \Omega_{2}$, and $N=40$ for regions $\Omega_{3}, \Omega_{4}$ ). The well-known singularity should be noted in Fig. $2(b)$, at the corner due to the discontinuous boundary condition (see [18] for more details). Also looking at external domain curves, we can notice that the gradient conditions (110) and (111) are met.

\section{B. Wave excitation force comparison - velocity potential vs Haskind's relation method}

A comparison is made between the two approaches presented in Section IV to evaluate the excitation forces. The first approach is based on the solution of the scattering problem and given by equation (153) and the other one is based on the Haskind's relation (156). Fig. 3, shows numerical results for case study No.2 with $\alpha=\left[\begin{array}{lll}2 & 1 & .51\end{array}\right]$. Excitation forces are non-dimensionalised dividing them by hydrostatic stiffness i.e. $\rho \mathrm{g} \pi\left(R_{b}^{2}-R_{c}^{2}\right)$ for the buoy, and $\rho \mathrm{g} \pi R_{c}^{2}$ for the submerged platform. Evidently, the two approaches obtain very well matched results, which implies that the correctness of the scattering potential seems to be confirmed.

\section{Asymptotic behaviour comparison with existing models}

Also one could use asymptotic behaviours of the presented model to compare results with existing models found in the literature, which indirectly verify the proposed expressions for potentials. Figures 4 to 9 show numerical results 


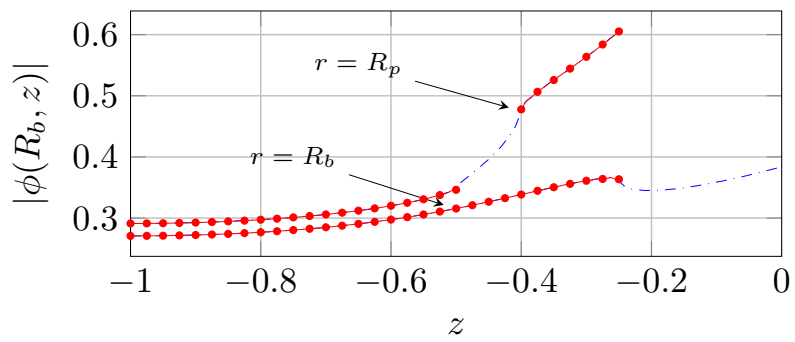

(a)

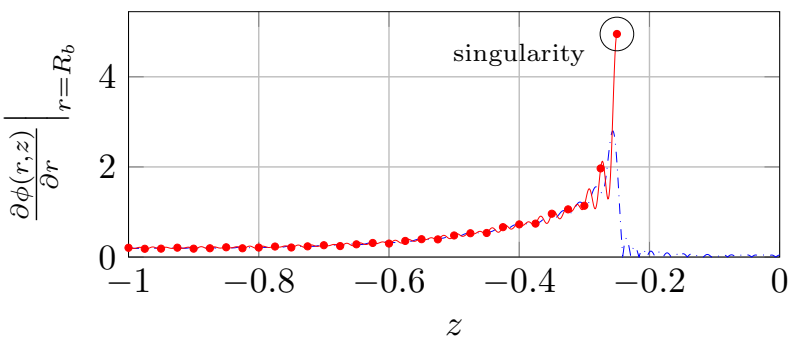

(b)

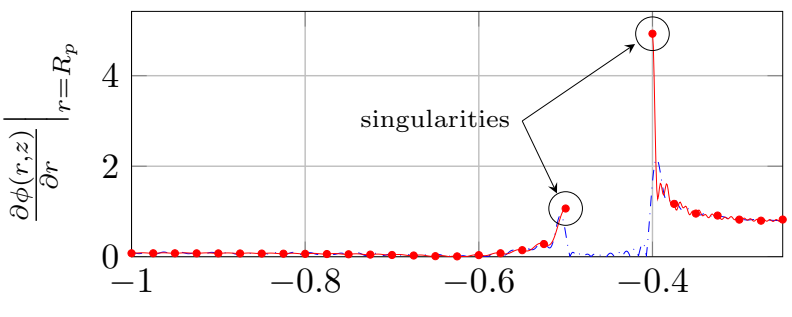

(c)

$\ldots$ Ext. domain $\longrightarrow$ Int. domain

Fig. 2. Matching of the velocity potential $(a)$ and its first derivative $(b)$ and $(c)$ for case study No.3, $\alpha=.75$, at the imaginary interface $r=R_{b}$ and $r=R_{p}$ for $k_{e}=1$.

for hydrodynamic parameters when the column radius tends towards zero, case study No.1 for $\alpha=\left[\begin{array}{lll}8 & 2 & 1\end{array}\right]$. This configuration is similar to the model presented by Wu et al. in [14] (not shown). Same coefficients as those given in [14] have been used to non-dimensionnalise excitation forces in Fig. 4 and hydrodynamics coefficients in Figures 5 to 8, and excellent correlation is found. Also parameters are shown for the case where the radius of the column is half of the radius of the buoy that corresponds to case study No.2. Looking at the added mass and radiation damping, we note that only the amplitude of the coefficients changes with the increase and decrease of the wet body surface but not the behaviour. If we look at the excitation forces on the buoy, the presence of the column does not seem to have an effect on it. However, we notice that for the platform, the behaviour is totally different at low frequency and we can observe a rebound which varies with the radius of the plate except for relatively big sizes. It seems that a radius ratio between the plate and column exists for which the column no longer influences the behaviour of the wave excitation force on the platform. In addition, a last check is performed on the correctness of 


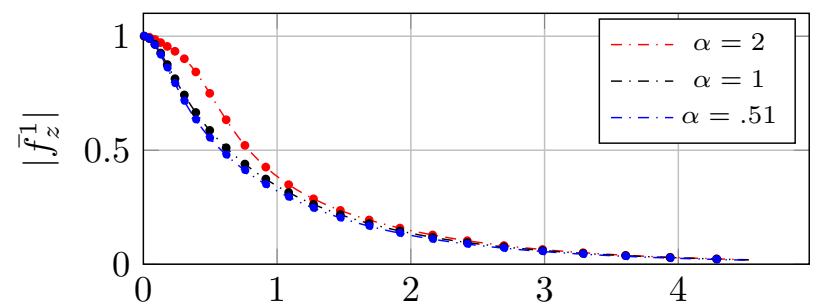

(a)

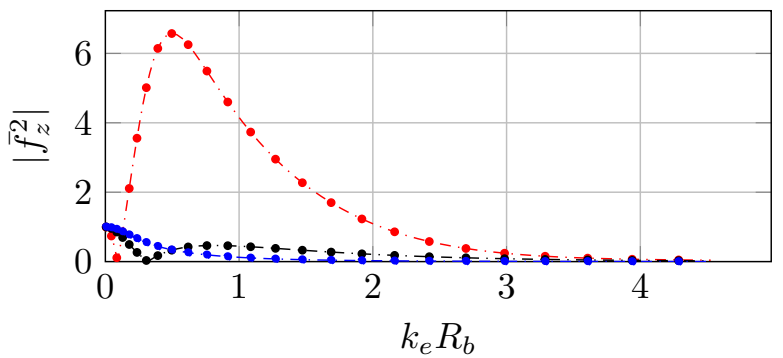

(b)

velocity potential • Haskind's relation

Fig. 3. Dimensionless vertical forces, $(a)$ on buoy $(b)$ on platform, for case study No.2.

numerical results, using the symmetry of the off-diagonal elements in the matrices for the added mass and radiation damping coefficients as illustrated in Fig. 9.

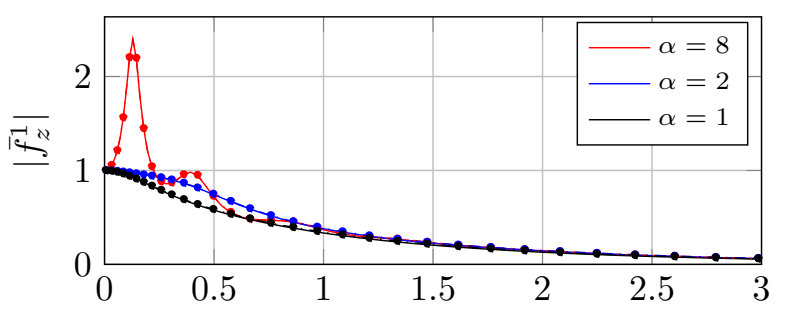

(a)

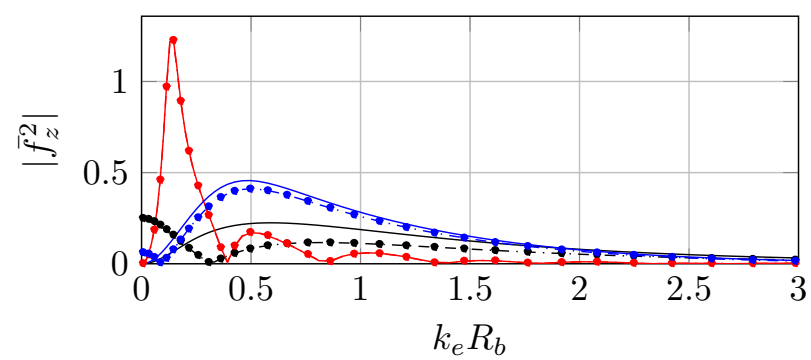

(b) case No.1 -.. case No.2

Fig. 4. Dimensionless vertical forces, $(a)$ on buoy $(b)$ on platform. 


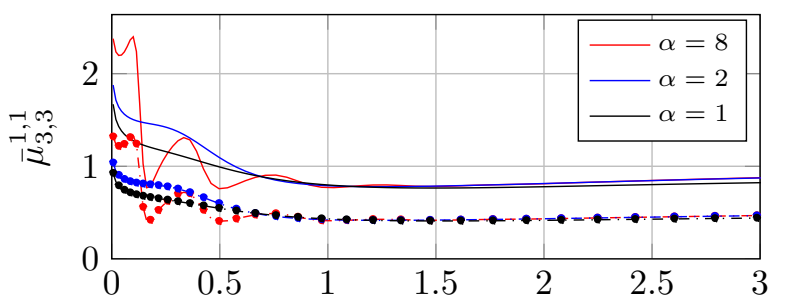

(a)

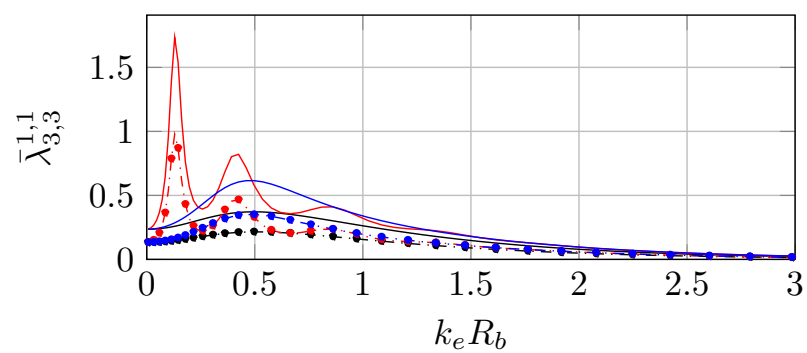

(b)

case No.1 - c. case No.2

Fig. 5. Dimensionless added mass $(a)$ and radiation damping $(b)$ for the buoy.

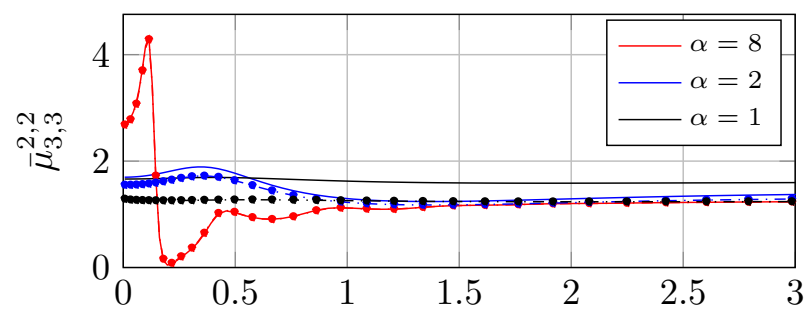

(a)

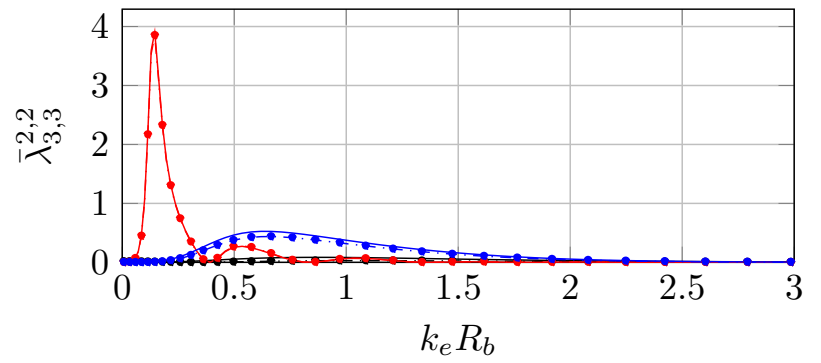

(b)

case No.1 -... case No.2

Fig. 6. Dimensionless added mass $(a)$ and radiation damping $(b)$ for the platform.

Figures 10 to 11 show numerical results for hydrodynamic parameters when plate radius tends toward column radius (case study No.3 for $\alpha=.51$ ) for two different water depths $h=1$ and $h=5$. This configuration is similar to the model presented by Chau and Yeung in [15]. The same coefficients as above have been used to 


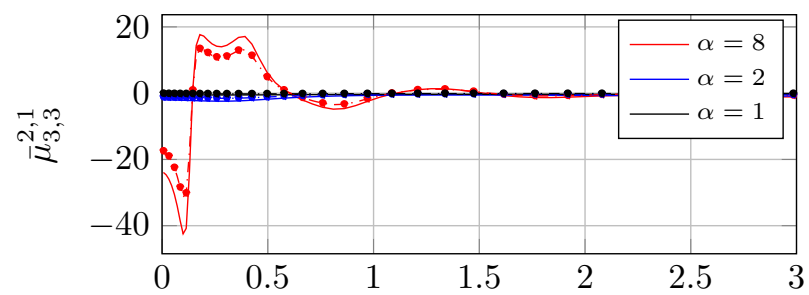

(a)

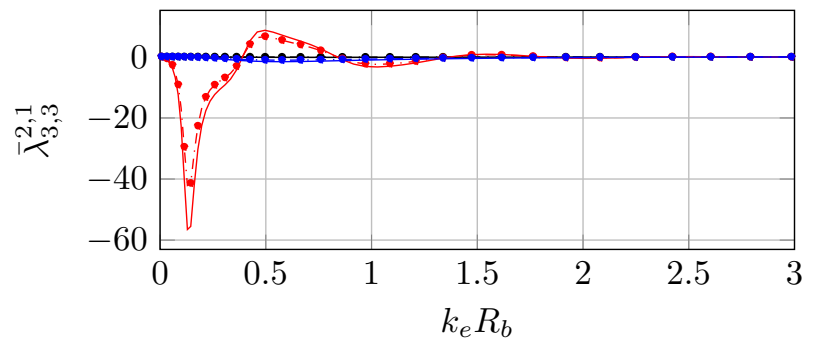

(b)

_ case No.1 _ _ case No.2

Fig. 7. Dimensionless added mass $(a)$ and radiation damping $(b)$ for the buoy due to the platform motion.

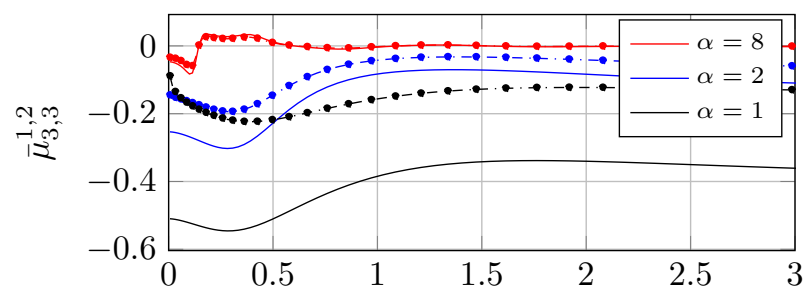

(a)

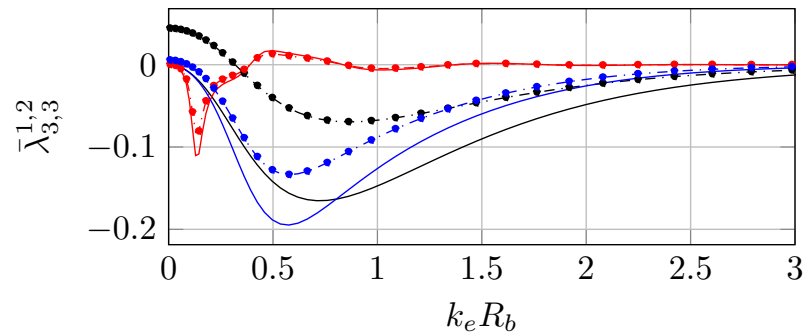

(b)

_ case No.1 _ . case No.2

Fig. 8. Dimensionless added mass $(a)$ and radiation damping $(b)$ for the platform due to the buoy motion. 


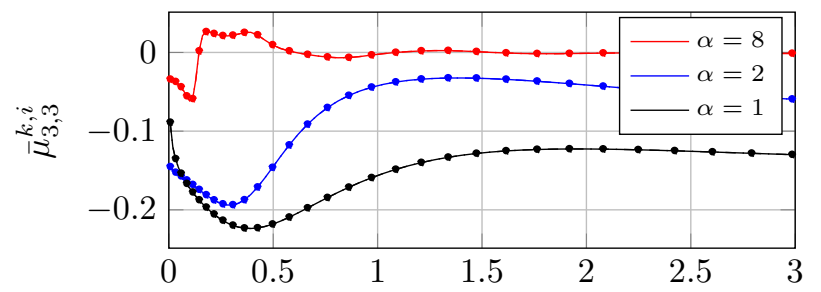

(a)

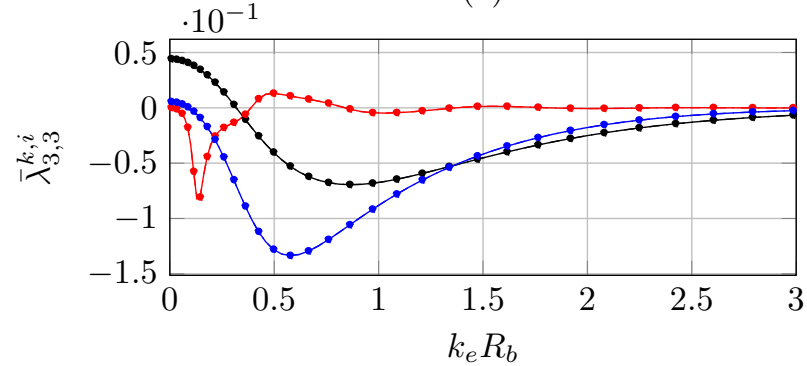

(b)

$\longrightarrow(k, i)=(1,2) \cdots(k, i)=(2,1)$

Fig. 9. Non-diagonal elements in the matrices for the added mass $(a)$ and radiation damping $(b)$ coefficients divide by $\frac{2}{3} \rho \mathrm{g} \pi R_{p}^{3}$ for case study No.2.

non-dimensionnalised excitation forces. For hydrodynamic coefficients, we divided by factor $\rho \pi R_{b}^{2}$ for the buoy and by factor $\rho \pi R_{c}^{2}$ for the platform. Here again figures show very good correlation between our model and the literature. Looking at the excitation forces on the platform, Fig. 10 (b), one can notice that the behaviour is not as previously described. It seems that a radius ratio between the plate and column exists for which the plate radius is not big enough for the rebound to be observed. Fig. 12 shows variation of the excitation force on the platform for different column and plate radiuses. Also we compare results with the case of a heaving single cylinder (dash-dotted lines) presented by Battha and Rahman in [7] with the same geometrical parameters as the column. When the radius ratio between plate and column is relatively small and the bearing surface of the plate is not big enough, it can be noted that the platform behaves in a similar way as a single cylinder. As already mentioned, for a relatively large plate radius compared to that of the column, it is found that the platform mainly behaves as the plate without the column. Between these two extremes, we have a rebound phenomenon for which properties such as amplitude and peak amplitude frequency vary with bearing surface.

\section{Conclusion}

Based on the potential theory, a semi-analytical method has been presented in order to solve the radiation and scattering problems which provides hydrodynamic parameters in heaving mode for a specific wave energy converter. These parameters are particularly needed for designers to analyse the WEC dynamics in irregular waves. A specific code has been developed based on the mathematical formulations presented in this paper. For validation purposes, several numerical simulations have been carried out for different buoy, column, and plate radiuses. The obtained 


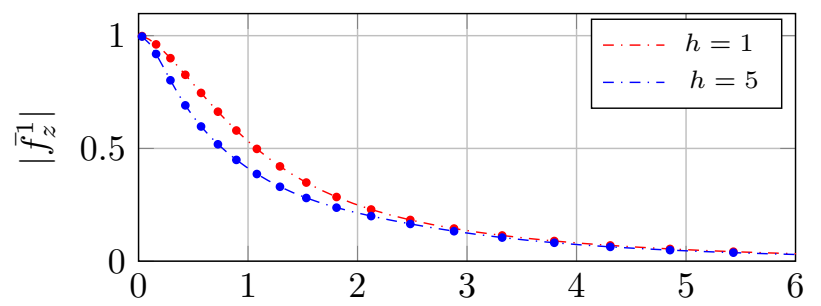

(a)

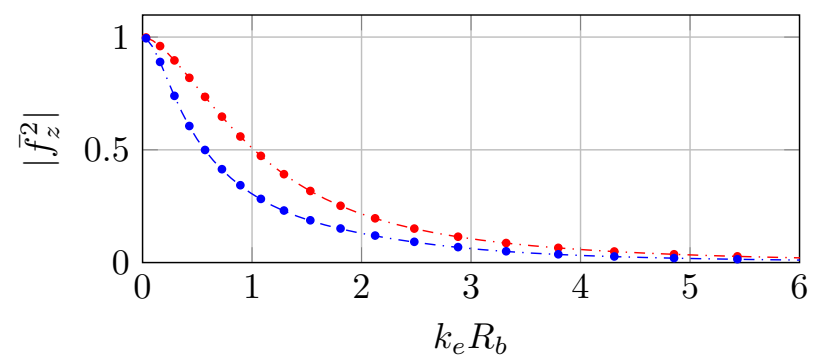

(b)

-..- $\alpha=.51 \cdot$ Chau and Yeung [15]

Fig. 10. Dimensionless vertical forces on the buoy $(a)$, on the platform $(b)$.

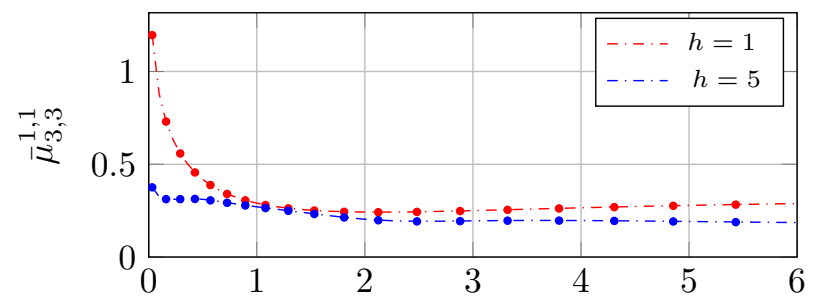

(a)

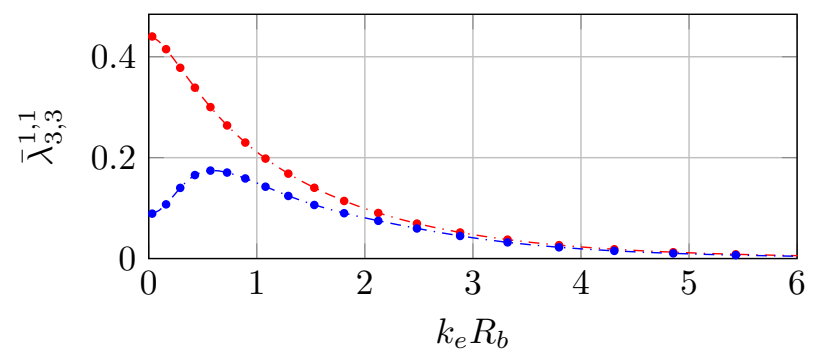

(b)

-.-. $\alpha=.51 \cdot$ Chau and Yeung [15]

Fig. 11. Dimensionless added mass $(a)$ and radiation damping $(b)$ for the buoy. 


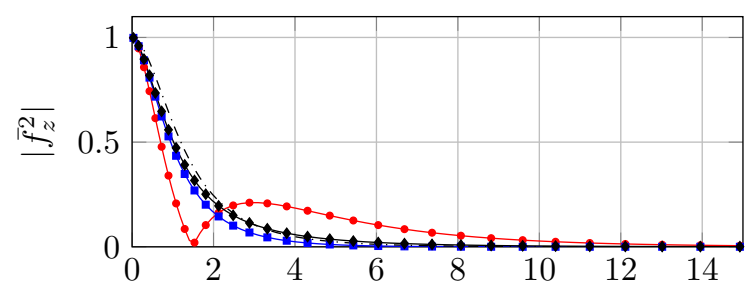

(a) $-\beta=.5$

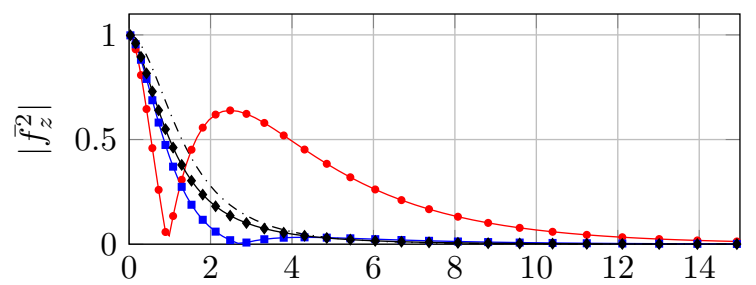

(b) $-\beta=.35$

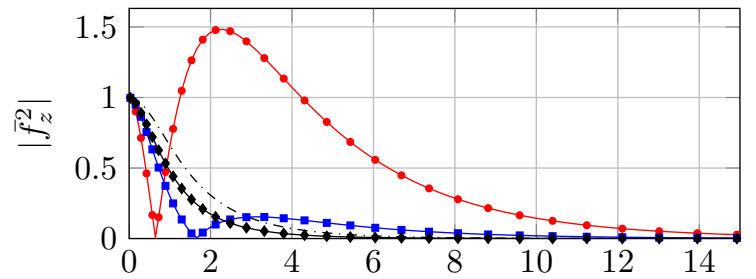

(c) $-\beta=.25$

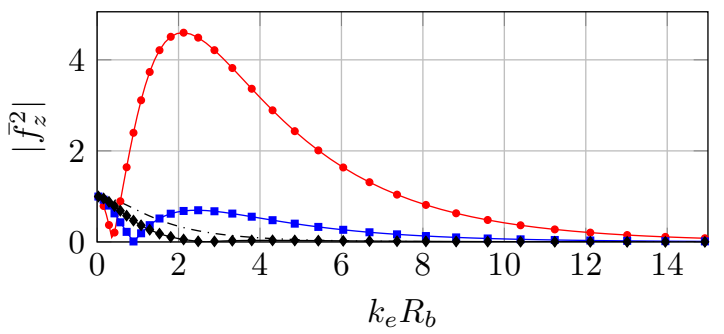

(d) $-\beta=.15$

$\because \alpha=1 \multimap \alpha=.75 \multimap \alpha=.51 \ldots$ one cylinder

Fig. 12. Dimensionless vertical forces on the platform for differents column and plate radius.

results have been compared to well-known and available models in the literature. These results clearly confirm the appropriateness of the proposed semi-analytical approach. Finally, a set of theoretical results for the wave excitation forces, added mass and radiation damping have been presented for different submerged plate to buoy radius ratio as well as column to buoy radius ratio. These results have obviously shown that excitation forces applied on the submerged platform have a remarkable behaviour that depends on the plate bearing surface for a given column radius. Regarding the available literature, this behaviour is the first reported for that kind of structure and future investigations will be carried out on the studied structure for wave energy extraction. 


\section{APPENDIX A}

EXPRESSION FOR $S_{l}(r), \tilde{S}_{l}(r)$

- In the case where $R_{b}<R_{p}$

for $l=0$

$$
\begin{aligned}
& S_{0}=\frac{H_{0}^{(1)}\left(k_{i} r\right) H_{0}^{(2)}\left(k_{i} R_{b}\right)-H_{0}^{(1)}\left(k_{i} R_{b}\right) H_{0}^{(2)}\left(k_{i} r\right)}{H_{0}^{(1)}\left(k_{i} R_{p}\right) H_{0}^{(2)}\left(k_{i} R_{b}\right)-H_{0}^{(1)}\left(k_{i} R_{b}\right) H_{0}^{(2)}\left(k_{i} R_{p}\right)} \\
& \tilde{S}_{0}=\frac{H_{0}^{(1)}\left(k_{i} R_{p}\right) H_{0}^{(2)}\left(k_{i} r\right)-H_{0}^{(1)}\left(k_{i} r\right) H_{0}^{(2)}\left(k_{i} R_{p}\right)}{H_{0}^{(1)}\left(k_{i} R_{p}\right) H_{0}^{(2)}\left(k_{i} R_{b}\right)-H_{0}^{(1)}\left(k_{i} R_{b}\right) H_{0}^{(2)}\left(k_{i} R_{p}\right)}
\end{aligned}
$$

for $l \geq 1$

$$
\begin{gathered}
S_{l}=\frac{I_{0}\left(\gamma_{l} r\right) K_{0}\left(\gamma_{l} R_{b}\right)-I_{0}\left(\gamma_{l} R_{b}\right) K_{0}\left(\gamma_{l} r\right)}{I_{0}\left(\gamma_{l} R_{p}\right) K_{0}\left(\gamma_{l} R_{b}\right)-I_{0}\left(\gamma_{l} R_{b}\right) K_{0}\left(\gamma_{l} R_{p}\right)} \\
\tilde{S}_{l}=\frac{I_{0}\left(\gamma_{l} R_{p}\right) K_{0}\left(\gamma_{l} r\right)-I_{0}\left(\gamma_{l} r\right) K_{m}\left(\gamma_{l} R_{p}\right)}{I_{0}\left(\gamma_{l} R_{p}\right) K_{0}\left(\gamma_{l} R_{b}\right)-I_{0}\left(\gamma_{l} R_{b}\right) K_{0}\left(\gamma_{l} R_{p}\right)}
\end{gathered}
$$

- In the case where $R_{b}>R_{p}$

for $l=0$

$$
S_{0}=\frac{\ln \left(r / R_{p}\right)}{\ln \left(R_{b} / R_{p}\right)} \quad \tilde{S}_{00}=\frac{\ln \left(R_{b} / r\right)}{\ln \left(R_{b} / R_{p}\right)}
$$

for $l \geq 1$

$$
\begin{gathered}
S_{l}=\frac{I_{0}\left(\gamma_{l} r\right) K_{0}\left(\gamma_{l} R_{p}\right)-I_{0}\left(\gamma_{l} R_{p}\right) K_{0}\left(\gamma_{l} r\right)}{I_{0}\left(\gamma_{l} R_{b}\right) K_{0}\left(\gamma_{l} R_{p}\right)-I_{0}\left(\gamma_{l} R_{p}\right) K_{0}\left(\gamma_{l} R_{b}\right)} \\
\tilde{S}_{l}=\frac{I_{0}\left(\gamma_{l} R_{b}\right) K_{0}\left(\gamma_{l} r\right)-I_{0}\left(\gamma_{l} r\right) K_{0}\left(\gamma_{l} R_{b}\right)}{I_{0}\left(\gamma_{l} R_{b}\right) K_{0}\left(\gamma_{l} R_{p}\right)-I_{0}\left(\gamma_{l} R_{p}\right) K_{0}\left(\gamma_{l} R_{b}\right)}
\end{gathered}
$$

\section{APPEndix B}

EXPRESSION FOR $T_{n}(r), \tilde{T}_{n}(r)$

for $n=0$

$$
T_{0}=\frac{\ln \left(r / R_{c}\right)}{\ln \left(R_{x} / R_{c}\right)} \quad \tilde{T}_{0}=\frac{\ln \left(R_{x} / r\right)}{\ln \left(R_{x} / R_{c}\right)}
$$

for $n \geq 1$

$$
\begin{aligned}
& T_{n}=\frac{I_{0}\left(\gamma_{l} r\right) K_{0}\left(\gamma_{l} R_{c}\right)-I_{0}\left(\gamma_{l} R_{c}\right) K_{0}\left(\gamma_{l} r\right)}{I_{0}\left(\gamma_{l} R_{x}\right) K_{0}\left(\gamma_{l} R_{c}\right)-I_{0}\left(\gamma_{l} R_{c}\right) K_{0}\left(\gamma_{l} R_{x}\right)} \\
& \tilde{T}_{n}=\frac{I_{0}\left(\gamma_{l} R_{x}\right) K_{0}\left(\gamma_{l} r\right)-I_{0}\left(\gamma_{l} r\right) K_{0}\left(\gamma_{l} R_{x}\right)}{I_{0}\left(\gamma_{l} R_{x}\right) K_{0}\left(\gamma_{l} R_{c}\right)-I_{0}\left(\gamma_{l} R_{c}\right) K_{0}\left(\gamma_{l} R_{x}\right)}
\end{aligned}
$$

where $R_{x}=\min \left(R_{b}, R_{p}\right)$. 


\section{ACKNOWLEDGMENT}

This work was supported in part by the Fonds Unique Interministériel (France) — Project "EM Bilboquet", in part by Région Bretagne, and in part by Conseil Général du Finistère.

\section{REFERENCES}

[1] J. V. Wehausen, "The Motion of Floating Bodies," Annual Review of Fluid Mechanics, vol. 3, no. 1, pp. 237-268, Jan. 1971.

[2] B. Molin, Hydrodynamique des structures offshore - Guides pratiques sur les ouvrages en mer, in French. Technip, 2002.

[3] S. Olaya, J.-M. Bourgeot, and M. Benbouzid, "Hydrodynamic Coefficients and Wave Loads for a WEC Device in Heaving Mode," in Proceedings of the 2013 MTS/IEEE OCEANS, Bergen (Norway), Jun. 2013, pp. 1-6.

[4] L. Berggren and M. Johansson, "Hydrodynamic coefficients of a wave energy device consisting of a buoy and a submerged plate," Applied Ocean Research, vol. 14, no. 1, pp. 51-58, Jan. 1992.

[5] R. W. Yeung, "Added mass and damping of a vertical cylinder in finite-depth waters," Applied Ocean Research, vol. 3, no. 3, pp. 119-133, Jul. 1981.

[6] C. J. R. Garrett, "Wave forces on a circular dock," Journal of Fluid Mechanics, vol. 46, no. 01, pp. 129-139, Mar. 1971.

[7] D. D. Bhatta and M. Rahman, "On scattering and radiation problem for a cylinder in water of finite depth," International Journal of Engineering Science, vol. 41, no. 9, pp. 931-967, May 2003.

[8] D. D. Bhatta, "Computations of hydrodynamic coefficients, displacement-amplitude ratios and forces for a floating cylinder due to wave diffraction and radiation," International Journal of Non-Linear Mechanics, vol. 46, no. 8, pp. 1027-1041, Oct. 2011.

[9] H. Eidsmoen, "Hydrodynamic parameters for a two-body axisymmetric system," Applied Ocean Research, vol. 17, no. 2, pp. 103-115, Jan. 1995.

[10] Y. Zheng, Y. Shen, Y. You, B. Wu, and L. Rong, "Hydrodynamic properties of two vertical truncated cylinders in waves," Ocean Engineering, vol. 32, no. 3-4, pp. 241-271, Mar. 2005.

[11] A. N. Williams, W. Li, and K.-H. Wang, "Water wave interaction with a floating porous cylinder," Ocean Engineering, vol. 27, no. 1, pp. 1-28, Jan. 2000.

[12] B.-J. Wu, Y.-H. Zheng, Y.-G. You, X.-Y. Sun, and Y. Chen, "On diffraction and radiation problem for a cylinder over a caisson in water of finite depth," International Journal of Engineering Science, vol. 42, no. 11-12, pp. 1193-1213, Jul. 2004.

[13] B.-J. Wu, Y.-H. Zheng, and Y.-G. You, "Response Amplitude and Hydrodynamic Force for a Buoy over a Convex," Journal of Waterway, Port, Coastal, and Ocean Engineering, vol. 132, no. 2, pp. 97-105, Mar. 2006.

[14] B.-J. Wu, Y.-H. Zheng, Y. You, D. Jie, and Y. Chen, "On diffraction and radiation problem for two cylinders in water of finite depth," Ocean Engineering, vol. 33, no. 5-6, pp. 679-704, Apr. 2006.

[15] F. P. Chau and R. W. Yeung, "Inertia, Damping, and Wave Excitation of Heaving Coaxial Cylinders," in 31st International Conference on Ocean, Offshore and Arctic Engineering. Rio de Janeiro, Brazil: ASME, Jul. 2012, pp. 803-813.

[16] C. Cochet and R. W. Yeung, "Two-Component Axisymmetric Wave-Energy Absorber - Analysis of Dynamics and Geometric Proportions," in The 27th International Workshop on Water Waves and Floating Bodies, Copenhagen, Denmark, 2012.

[17] S. A. Mavrakos, "Hydrodynamic coefficients in heave of two concentric surface-piercing truncated circular cylinders," Applied Ocean Research, vol. 26, no. 3-4, pp. 84-97, May 2004.

[18] C. Linton and P. McIver, Handbook of Mathematical Techniques for Wave/Structure Interactions, 1st ed. Chapman and Hall/CRC, 2001.

[19] J. Falnes, Ocean Waves and Oscillating Systems - Linear Interactions Including Wave-Energy Extraction. Cambridge University Press, Apr. 2002.

[20] M. D. Haskind, "The Exciting Forces and Wetting of Ships in Waves," David Taylor Model Basin, Tech. Rep., Nov. 1962.

[21] J. N. Newman, "The exciting forces on fixed bodies in waves," David Taylor Model Basin, Tech. Rep., May 1963. 


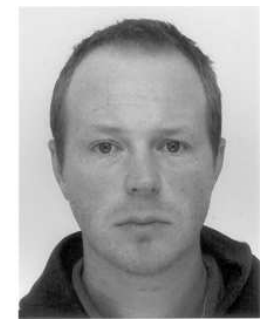

Sébastien Olaya Sébastien Olaya was born in Brest, France in 1986. He received his M.Sc. degree in electronic engineering, from the École Nationale d'Ingénieurs de Brest, Brest, France and his M.Sc. degree in control systems engineering, from the Université de Bordeaux I, Bordeaux, France, respectively in 2009 and 2011. He is currently working towards his Ph.D. degree on wave energy converter control at the École Nationale d'Ingénieurs de Brest.

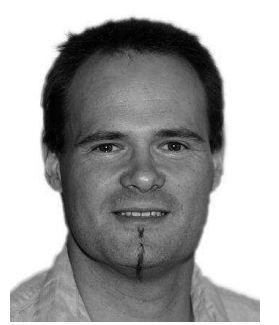

Jean-Matthieu Bourgeot Jean-Matthieu Bourgeot was born in Grenoble, France in 1977. He received his Ph.D. degree in control systems engineering from the National Polytechnic Institute of Grenoble, Grenoble, France, in 2004. He is currently an Associate Professor of control engineering at the Department of Mechatronics of the École Nationale d'Ingénieurs de Brest, Brest, France. His current research interests are control and diagnosis of non-linear systems and shape memory actuators control.

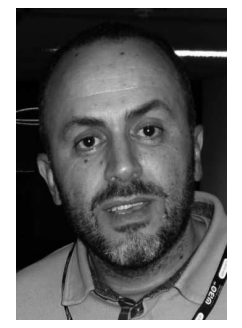

Mohamed El Hachemi Benbouzid Mohamed El Hachemi Benbouzid (S92-M95-SM98) was born in Batna, Algeria, in 1968. He received his B.Sc. degree in electrical engineering from the University of Batna, Batna, Algeria, in 1990, his M.Sc. and Ph.D. degrees in electrical and computer engineering from the National Polytechnic Institute of Grenoble, Grenoble, France, in 1991 and 1994, respectively, and the Habilitation à Diriger des Recherches degree from the University of Picardie Jules Verne, Amiens, France, in 2000. After receiving his Ph.D. degree, he joined the Professional Institute of Amiens, University of Picardie Jules Verne, where he was an Associate Professor of electrical and computer engineering. In September 2004, he joined the University Institute of Technology (IUT) of Brest, University of Brest, Brest, France, as a Professor of electrical engineering. His main research interests and experience include analysis, design, and control of electric machines, variable-speed drives for traction, propulsion, and renewable energy applications, and fault diagnosis of electric machines.

Prof. Benbouzid is an IEEE Senior Member. He is the Editor-in-Chief of the International Journal on Energy Conversion (IRECON). He is also an Associate Editor of the IEEE Transactions on Energy Conversion, the IEEE Transactions on Industrial Electronics, the IEEE Transactions on Sustainable Energy, and the IEEE Transactions on Vehicular Technology. He was an Associate Editor of the IEEE/ASME Transactions on Mechatronics from 2006 to 2009. 